\title{
Prediction of airborne sound and impact sound insulation provided by single and multilayer systems using analytical expressions
}

\author{
A. Tadeu* , A. Pereira, L. Godinho, J. António \\ Department of Civil Engineering, Pólo II of the University of Coimbra, Rua Luís Reis Santos, \\ 3030-788 Coimbra, Portugal
}

Received 25 May 2006; accepted 26 May 2006

Available online 17 July 2006

\begin{abstract}
In this work, the authors use analytical solutions to assess the airborne sound and impact insulation provided by homogeneous partitions that are infinite along their plane. The algorithm uses Green's functions, derived on the basis of previous work by the authors on the prediction of airborne sound insulation provided by single and double panels. The model is now extended to handle multilayer systems, allowing the simulation of three-dimensional loads applied in both the acoustic and solid media.

The model is validated against experimental results and compared with simplified expressions for single, double and triple panels. The results provided by the analytical model were found to provide a good agreement with the experimental results, except in the vicinity of the coincidence effect in the presence of thicker panels.

The applicability of the proposed tool is then illustrated by analyzing the acoustic behavior provided by single layers and by a suspended ceiling. Different variables are studied, such as the mass, the stiffness of the layers, the position and direction of the load within the elastic medium and the presence of porous material in the fluid layers. It was found that the model is able to simulate the acoustic phenomena involved in single and multilayer systems.
\end{abstract}

(c) 2006 Elsevier Ltd. All rights reserved.

Keywords: Airborne; Impact; Multilayer; Experimental

\footnotetext{
${ }^{*}$ Corresponding author. Tel.: +351 239797201; fax: +351 239797190.

E-mail address: tadeu@dec.uc.pt (A. Tadeu).
} 


\section{Introduction}

The transmission of airborne sound energy through a single separation element depends on several variables, such as the frequency of sound incident on the element, the physical properties of the panel (mass, internal damping, elasticity modulus, Poisson's ratio), the connections with the surrounding structure and the vibration eigenmodes of the element. The prediction of the physical phenomena regarding wave propagation is quite complex, and this has led to several simplified models such as the theoretical Mass Law, which assumes the element behaves like a group of infinite juxtaposed masses with independent displacement and null damping forces. Sewell [1] and Sharp [2] have proposed other simplified models for the frequencies below, in the vicinity of and above the coincidence effect to predict the airborne sound insulation provided by single panels.

But predicting the dynamic behavior of a multilayer system turns out to be more complex. Different simplified approaches have been proposed over the years. London [3] predicted the sound insulation provided by double walls. In this model the double wall is excited by plane waves at frequencies below the critical frequency and the mass is controlled to disregard panel resonance. The equation proposed by London takes into account the effect of the resonance which occurs within the air gap. Beranek [4] later performed some mathematical manipulations in order to consider mass-air-mass resonance. The effect of an air gap filled with a porous sound-absorbing material has been simulated by other authors [5].

Fringuellino et al. [6] calculated the transmission loss in multi-layered walls using a simplified approach based on the prior knowledge of the characteristic impedance of each material layer. Bolton et al. [7] described a theory for multi-dimensional wave propagation in elastic porous material, based on Biot's theory, and used it to predict the airborne sound insulation provided by foam-lined panels. When aluminium double-panel structures lined with polyurethane foam were studied, the results provided by their models were found to be good when compared with experimental results.

It is also important to predict the impact sound insulation provided by partitions at the design stage. The development of a prediction model has to take the excitation and the sound transmission system into account. In dwellings, the sources of annoyance can be footsteps or the impact of dropped objects. To evaluate the impact sound level experimentally, a standardized tapping machine, as described in the ISO standards, is used. Although this machine does not simulate real footsteps, the test results yield important information concerning the dynamic behavior of the floor. Several authors have addressed the problem of the excitation source, where the interaction at the interfaces between the hammer and the floor has to be considered. Cremer [8] has derived an impact source spectrum caused by the tapping machine acting on homogeneous floors of high impedance. He assumes that the impact is perfectly elastic and the results were proved to be satisfactory for several frequencies. Vér [9] derived a complete description of the force spectrum and impact level provided by the tapping machine on hard surfaces. He also considered the improvement in insulation provided by the use of elastic surface layers or by floating floors with high-impedance surfaces.

Although the final response provided by the loads that act in the acoustic or in the elastic medium differs, the dynamic behavior of partitions may present similarities. Heckl et al. [10] found a relation between the airborne and impact sound insulation provided by partitions. 
In this work, the authors propose an analytical model to assess the acoustic behavior of single or multilayer partitions, infinite along their plane, dividing an infinite acoustic medium. The formulation of a set of analytical solutions for calculating the acoustic insulation provided by single and double walls when submitted to incident pressure fields has been presented in earlier work [11-13]. This paper generalizes that model to solve structures with an arbitrary number of elastic and acoustic layers, also allowing the application of three-dimensional impact loads. Thus, the proposed model can be used to predict the acoustic behavior of a broader range of acoustic systems than other models found in the literature. In addition, it overcomes certain restrictions, such as the type and position of the load, the number of layers and the assumption of the existence of incident plane waves. However, the model described here does not take into account the presence of flanking transmissions or sound bridges, since it assumes that the panels are uniform layers of infinite extent, without mechanical fixings.

The present model defines a set of potentials in each layer that are combined so as to verify the boundary conditions and to predict the three-dimensional airborne and impact sound insulation (vertically and horizontally) provided by either a single structural layer or by a multilayer system. A point load is first represented by a summation of two-dimensional linear loads after the application of a Fourier transformation along the $z$ direction (2.5D problem). Each linear load is in turn modeled as a superposition of plane sources following the application of an additional Fourier transformation in the $x$ direction.

The following section outlines the analytical 3D and $2.5 \mathrm{D}$ formulations used to predict airborne and impact sound insulation. The analytical formulation is then validated with experimental results and compared with simplified expressions referenced in the literature review. For this, the airborne sound insulation provided by single and double panels is discussed, after which the impact sound insulation provided by a single panel and by a floating layer system is examined. Finally, the applicability of the model is determined by assessing the acoustic behavior provided by a single structural layer excited by point loads and by a suspended ceiling.

\section{Analytical solution formulation}

The analytical solutions are derived in the frequency domain for a multilayer system, infinite along the $x$ and $z$ directions, dividing an infinite acoustic medium (see Fig. 1). This system can combine several elastic and fluid layers with different thicknesses $h_{j}$ $(j=1,2, \ldots, n$ identifies the number of the layers).

The material properties and the wave velocities allowed in each individual panel are defined as in Table 1.

\subsection{Formulation of the $3 D$ problem}

Consider the above-defined model to be excited by a point load, acting within one of its layers. For the case of point pressure loads acting in a fluid layer at $\left(x_{0}, y_{0}, z_{0}\right)$, the incident pressure wave field can be expressed by

$$
\sigma^{\mathrm{full}}(\omega, x, y, z)=\frac{A \mathrm{e}^{\mathrm{i} \frac{\omega}{j}\left(c^{j} t-\sqrt{\left(x-x_{0}\right)^{2}+\left(y-y_{0}\right)^{2}+\left(z-z_{0}\right)^{2}}\right)}}{\sqrt{\left(x-x_{0}\right)^{2}+\left(y-y_{0}\right)^{2}+\left(z-z_{0}\right)^{2}}}
$$




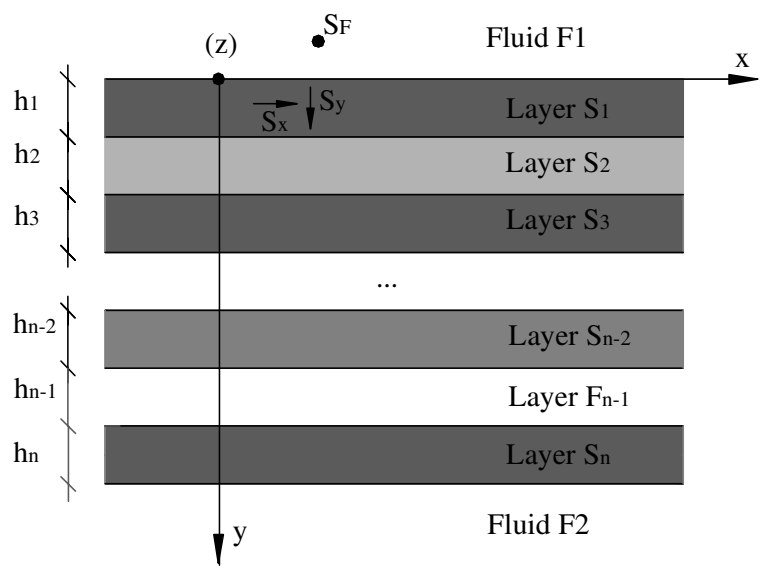

Fig. 1. Geometry of the problem.

Table 1

Material properties and wave velocities allowed in each individual panel

\begin{tabular}{ll}
\hline Fluid medium & Elastic medium \\
\hline Density $\rho_{f}^{j}$ & Density $\rho_{j}$ \\
Lamé constant $\lambda_{f}^{j}$ & Young's modulus $E_{j}$ \\
Dilatational wave velocity $c^{j}=\sqrt{\lambda_{f}^{j} / \rho_{f}^{j}}$ & Poisson's ratio $v_{j}$ \\
& Compressional wave velocity $c_{L}^{j}=\sqrt{\frac{E^{j}\left(1-v^{j}\right)}{\rho^{j}\left(1-2 v^{j}\right)\left(1+v^{j}\right)}}$ \\
& Shear wave velocity $c_{S}^{j}=\sqrt{\frac{E^{j}}{2 \rho^{j}\left(1+v^{j}\right)}}$ \\
\hline
\end{tabular}

in which $A$ is the wave amplitude and $\mathrm{i}=\sqrt{-1}$ and $\omega$ is the frequency of excitation. Assuming that the geometry of the problem does not change along the $z$ direction, when a Fourier transformation is applied along that direction [11], which is held to have an infinite number of virtual sources placed along it at equal intervals, $L$, then the incident pressure wave field, $\sigma^{\text {full }}(\omega, x, y, z)$, can be computed as a summation of two-dimensional sources,

$$
\sigma^{\text {full }}(\omega, x, y, z)=\frac{2 \pi}{L} \sum_{m=-\infty}^{\infty} \hat{\sigma}^{\text {full }}\left(\omega, x, y, k_{z}\right) \mathrm{e}^{-\mathrm{i} k_{z} z}
$$

where

$$
\hat{\sigma}^{\text {full }}\left(\omega, x, y, k_{z}\right)=\frac{-\mathrm{i} A}{2} H_{0}^{(2)}\left(k_{c}^{j} \sqrt{\left(x-x_{0}\right)^{2}+\left(y-y_{0}\right)^{2}}\right) \mathrm{e}^{-\mathrm{i} k_{z} z}
$$

in which $k_{c}^{j}=\sqrt{\omega^{2} /\left(c^{j}\right)^{2}-k_{z}^{2}}$ (with $\left.\operatorname{Im} k_{c}^{j}<0\right), k_{z}$ is the spatial wavenumber along the $z$ direction $\left(k_{z}=\frac{2 \pi}{L} m\right)$ and $H_{n}^{(2)}(\ldots)$ are second Hankel functions of order $n$.

This equation converges and can be approximated by a finite sum of terms. The distance $L$ needs to be large enough to avoid spatial contamination. The use of complex frequencies further reduces the influence of the neighbouring fictitious sources.

Using this technique, the scattered field caused by a point pressure load in the presence of the layered system can likewise be obtained as a discrete summation of 2D harmonic 
line loads, with different values of $k_{z}$. This problem is often referred to in the literature as a $2.5 \mathrm{D}$ problem, because the geometry is $2 \mathrm{D}$ and the source is $3 \mathrm{D}$. The same procedure can be applied to point loads acting in the $x$ and $y$ directions, in a solid medium, allowing those problems to be solved as discrete summations of simpler 2.5D problems [11].

\subsection{Formulation of the $2.5 \mathrm{D}$ problems}

In this work, a generalization of the technique proposed by Tadeu et al. [11-13] is performed in order to handle multilayer systems and the application of impact loads. The referenced authors derived a procedure to calculate the pressure field generated by a single panel, based on knowing the solid layer displacement potentials and the pressure potentials due to excitation by a spatially varying harmonic line load. In that method the potentials are written as a superposition of plane waves, by means of a discrete wavenumber representation (after applying a Fourier transform in the $x$ direction). The integrals of the expressions are then transformed into a discrete summation by assuming an infinite number of plane sources distributed along the $x$ direction at equal intervals, $L_{x}$. It is then possible to compute the 2.5D response of the system as a summation of the effects of the defined plane sources. After computing the solutions for a full sequence of $2.5 \mathrm{D}$ problems with varying values of $k_{z}$, the full 3D field generated by point loads can be determined as a discrete summation of these $2.5 \mathrm{D}$ problems.

A multilayer system consists of a combination of solid and fluid layers. Thus, to achieve the solution provided by this system a set of dilatational and shear potentials generated at each solid/fluid interface (interface $a$ and $b$ ) must be defined. In a fluid layer (see Fig. 2a) the full description of the pressure field requires the knowledge of one dilatational potential at each interface, while in the solid layer (see Fig. 2b and c) the displacement field is computed by making use of one dilatational and two shear displacement potentials at each interface, which depend on the orientation of the applied load. Table 2 lists the full set of potentials.

In the expressions listed in Table 2 the coefficients correspond to: $E_{f}^{a, j}=\mathrm{e}^{-\mathrm{i} v_{n f}^{j}\left|y-y^{a, j}\right|}$; $E_{f}^{b, j}=\mathrm{e}^{-\mathrm{i} v_{n f}^{j}\left|y-y^{b, j}\right|} ; v_{n f}^{j}=\sqrt{\left(k_{p_{f}}^{j}\right)^{2}-k_{z}^{2}-k_{n}^{2}}$, where $\operatorname{Im}\left(v_{n f}^{j}\right) \leqslant 0 ; k_{p_{f}}^{j}=\omega / c^{j} ; y^{a, j}$ and $y^{b, j}$ are the $y$ coordinates of the interfaces $a$ and $b$ which define the layer $j$ using the coordinate system according to Fig. $1 ; \quad E_{b}^{a, j}=\mathrm{e}^{-\mathrm{i} v_{n}^{j}\left|y-y^{a, j}\right|} ; \quad E_{b}^{b, j}=\mathrm{e}^{-\mathrm{i} v_{n}^{j}\left|y-y^{b, j}\right|} ; \quad E_{c}^{a, j}=\mathrm{e}^{-\mathrm{i} \gamma_{n}^{j}\left|y-y^{a, j}\right|}$; $E_{c}^{b, j}=\mathrm{e}^{-\mathrm{i} \gamma_{n}^{j}\left|y-y^{b, j}\right|} ; \quad \gamma_{n}^{j}=\sqrt{\left(k_{s}^{j}\right)^{2}-k_{z}^{2}-k_{n}^{2}} ; \quad k_{s}^{j}=\omega / c_{S}^{j} ; \quad v_{n}^{j}=\sqrt{\left(k_{p}^{j}\right)^{2}-k_{z}^{2}-k_{n}^{2}}, \quad$ with $\operatorname{Im}\left(v_{n}^{j}\right) \leqslant 0 ; k_{p}^{j}=\omega / c_{L}^{j} ; E_{a}^{j}=\frac{1}{2 \rho^{j} \omega^{2} L_{x}} ; E_{d}=\mathrm{e}^{-\mathrm{i} k_{n}\left(x-x_{0}\right)} ; k_{n}=\frac{2 \pi}{L_{x}} n$ and $(x, y)$ defining the coordinates of a point inside the layer $j$. The coefficients $A_{n}^{x, S_{j}}, \ldots, F_{n}^{x, S_{j}}, A_{n}^{y, S_{j}}, \ldots, F_{n}^{y, S_{j}}, G_{n}^{F_{j}}$, and $H_{n}^{F_{j}}$ are unknowns which are determined by solving a system of equations defined for the specific multilayer problem. This system can be written by combining individual systems of equations that are established for each layer. Each individual system of equations is built by deriving the potentials in Table 2 in order to write the stresses and displacements at the surfaces according to the layer and the source applied. The individual systems of equations are fully described in Appendix 1. The final system of equations can then be written by combining these individual systems for each layer and prescribing the boundary conditions:

- at the solid/fluid interfaces: the continuity of normal displacements and stresses and null tangential stresses (corresponding to 4 equations); 

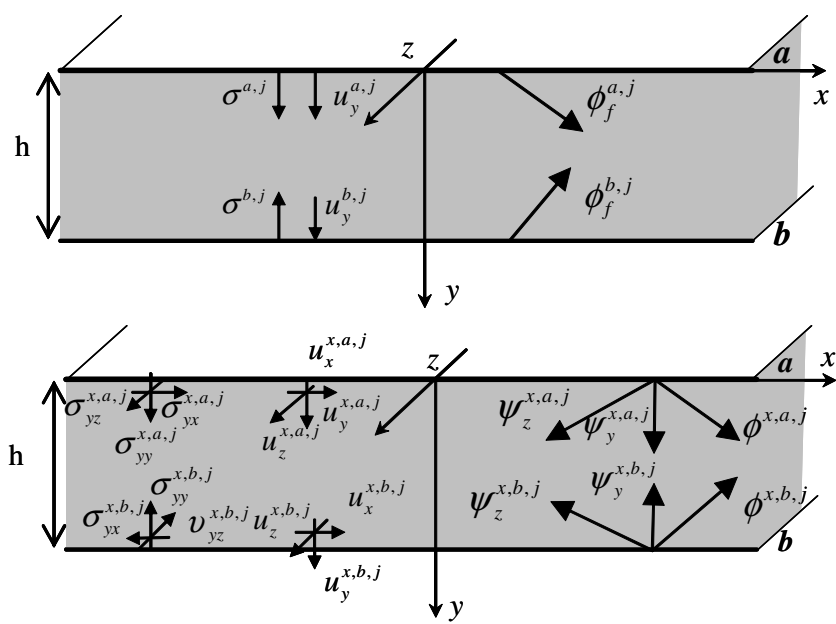

(a)

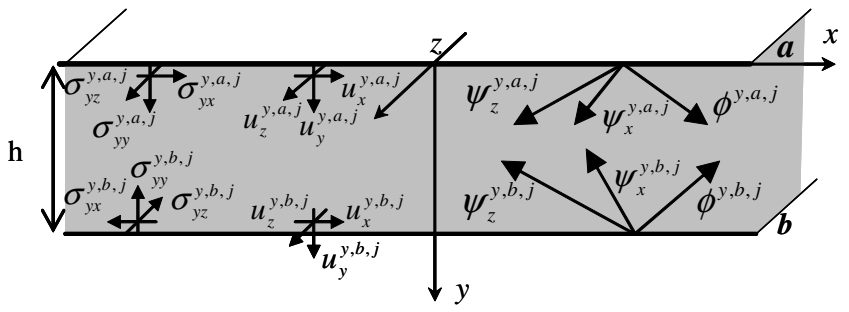

(b)

(c)

Fig. 2. Definition of potentials, stresses and displacements at the interfaces: (a) fluid layer; (b) solid layer when load acts in the $x$ direction; (c) solid layer when load acts in the $y$ direction.

- at the elastic/elastic interfaces: the continuity of displacements and stresses (corresponding to 6 equations);

- at the fluid/fluid interfaces: the continuity of normal displacements and pressures (corresponding to 2 equations).

Appendix 2 contains the definition of the final system of equations, assuming a set of elastic $\left(S_{j}\right.$ with $\left.j=1, \ldots, n\right)$ and fluid layers $\left(F_{j}\right.$ with $\left.j=1, \ldots, n\right)$, according to the multilayer system defined in Fig. 1, in order to illustrate how the final system of equations is built, assuming that a pressure load placed in Fluid 1 excites it.

The solution of this system will allow the unknown parameters to be determined and the scattered field at each medium can be calculated according to:

- Pressure field $\sigma_{j}$ at a point $(x, y)$ in a fluid layer $j$ :

$$
\sigma^{j}=-\frac{\mathrm{i}}{L_{x}} \sum_{n=-N}^{n=+N}\left(\frac{E_{f}^{a, j}}{v_{n f}^{j}} G_{n}^{F_{j}}\right) E_{d}-\frac{\mathrm{i}}{L_{x}} \sum_{n=-N}^{n=+N}\left(\frac{E_{f}^{b, j}}{v_{n f}^{j}} H_{n}^{F_{j}}\right) E_{d} .
$$

- Displacements $u_{d}^{x, j}$ (with $\left.d=x, y, z\right)$ at a point $(x, y)$ in an elastic layer $j$ when the load acts in the $x$ direction: 
Table 2

Potentials generated at each layer

\section{Fluid layer}

Pressure load/load acting in the elastic medium Interface (a)

$\phi_{f}^{a, j}=-\frac{\mathrm{i}}{L_{x}} \sum_{n=-N}^{n=+N}\left[\left(\frac{-\left(c^{j}\right)^{2}}{\omega^{2} \lambda_{f}^{j}}\right) \frac{E_{f}^{a, j}}{v_{n f}^{j}} G_{n}^{F_{j}}\right] E_{d}$

$$
\begin{aligned}
& \phi^{x, a, j}=E_{a}^{i} \sum_{n=-N}^{n=+N}\left(\frac{k_{n}}{v_{n}^{j}} E_{b}^{a, j} A_{n}^{x, S_{j}}\right) E_{d} \\
& \psi_{y}^{x, a, j}=E_{a}^{j} k_{z} \sum_{n=-N}^{n=+N}\left(\frac{E_{c}^{a, j}}{\gamma_{n}^{j}} B_{n}^{x, S_{j}}\right) E_{d} \\
& \psi_{z}^{x, a, j}=-E_{a}^{j} \sum_{n=-N}^{n=+N}\left(E_{c}^{a, j} C_{n}^{x, S_{j}}\right) E_{d}
\end{aligned}
$$

Elastic layer

Load acting in the $x$ direction

Load acting in the $y$ direction/pressure load

$$
\begin{aligned}
& \phi^{y, a, j}=E_{a}^{j} \sum_{n=-N}^{n=+N}\left(E_{b}^{a, j} A_{n}^{y, S_{j}}\right) E_{d} \\
& \psi_{x}^{y, a, j}=E_{a}^{j} k_{z} \sum_{n=-N}^{n=+N}\left(\frac{-E_{c}^{a, j}}{\gamma_{n}^{j}} C_{n}^{y, S_{j}}\right) E_{d} \\
& \psi_{z}^{y, a, j}=E_{a}^{j} \sum_{n=-N}^{n=+N}\left(\frac{k_{n}}{\gamma_{n}^{j}} E_{c}^{a, j} B_{n}^{y, S_{j}}\right) E_{d}
\end{aligned}
$$

Interface ( $b$ )

$\phi_{f}^{b, j}=-\frac{\mathrm{i}}{L_{x}} \sum_{n=-N}^{n=+N}\left[\left(\frac{-\left(c^{j}\right)^{2}}{\omega^{2} \lambda_{f}^{j}}\right) \frac{E_{f}^{b, j}}{v_{n f}^{j}} H_{n}^{F_{j}}\right] E_{d}$

$$
\begin{aligned}
& \phi^{x, b, j}=E_{a}^{j} \sum_{n=-N}^{n=+N}\left(\frac{k_{n}}{v_{n}^{j}} E_{b}^{b, j} D_{n}^{x, S_{j}}\right) E_{d} \\
& \psi_{y}^{x, b, j}=E_{a}^{j} k_{z} \sum_{n=-N}^{n=+N}\left(\frac{E_{c}^{b, j}}{\gamma_{n}^{j}} E_{n}^{x, S_{j}}\right) E_{d} \\
& \psi_{z}^{x, b, j}=E_{a}^{j} \sum_{n=-N}^{n=+N}\left(E_{c}^{b, j} F_{n}^{x, S_{j}}\right) E_{d}
\end{aligned}
$$$$
\phi^{y, b, j}=-E_{a}^{j} \sum_{n=-N}^{n=+N}\left(E_{b}^{b, j} D_{n}^{y, S_{j}}\right) E_{d}
$$$$
\psi_{x}^{y, b, j}=E_{a}^{j} k_{z} \sum_{n=-N}^{n=+N}\left(\frac{-E_{c}^{b, j}}{\gamma_{n}^{j}} F_{n}^{y, S_{j}}\right) E_{d}
$$$$
\psi_{z}^{y, b, j}=E_{a}^{j} \sum_{n=-N}^{n=+N}\left(\frac{k_{n}}{\gamma_{n}^{j}} E_{c}^{b, j} E_{n}^{y, j}\right) E_{d}
$$ 


$$
\begin{aligned}
u_{x}^{x, j}= & E_{a}^{j} \sum_{n=-N}^{n=+N}\left(\frac{-\mathrm{i} k_{n}^{2}}{v_{n}^{j}} E_{b}^{a, j} A_{n}^{x, S_{j}}-\frac{\mathrm{i} k_{z}^{2}}{\gamma_{n}^{j}} E_{c}^{a, j} B_{n}^{x, S_{j}}-\mathrm{i} \gamma_{n}^{j} E_{c}^{a, j} C_{n}^{x, S_{j}}\right) E_{d} \\
& +E_{a}^{j} \sum_{n=-N}^{n=+N}\left(\frac{-\mathrm{i} k_{n}^{2}}{v_{n}^{j}} E_{b}^{b, j} D_{n}^{x, S_{j}}-\frac{\mathrm{i} k_{z}^{2}}{\gamma_{n}^{j}} E_{c}^{b, j} E_{n}^{x, S_{j}}-\mathrm{i} \gamma_{n}^{j} E_{c}^{b, j} F_{n}^{x, S_{j}}\right) E_{d}, \\
u_{y}^{x, j}= & E_{a}^{j} \sum_{n=-N}^{n=+N}\left(-\mathrm{i} k_{n} E_{b}^{a, j} A_{n}^{x, S_{j}}+\mathrm{i} k_{n} E_{c}^{a, j} C_{n}^{x, S_{j}}\right) E_{d} \\
& -E_{a}^{j} \sum_{n=-N}^{n=+N}\left(-\mathrm{i} k_{n} E_{b}^{b, j} D_{n}^{x, S_{j}}+\mathrm{i} k_{n} E_{c}^{b, j} F_{n}^{x, S_{j}}\right) E_{d}, \\
u_{z}^{x, j}= & E_{a}^{j} \sum_{n=-N}^{n=+N}\left(\frac{-\mathrm{i} k_{z} k_{n}}{v_{n}^{j}} E_{b}^{a, j} A_{n}^{x, S_{j}}+\frac{\mathrm{i} k_{z} k_{n}}{\gamma_{n}^{j}} E_{c}^{a, j} B_{n}^{x, S_{j}}\right) E_{d} \\
& +E_{a}^{j} \sum_{n=-N}^{n=+N}\left(\frac{-\mathrm{i} k_{z} k_{n}}{v_{n}^{j}} E_{b}^{b, j} D_{n}^{x, S_{j}}+\frac{\mathrm{i} k_{z} k_{n}}{\gamma_{n}^{j}} E_{c}^{b, j} E_{n}^{x, S_{j}}\right) E_{d} .
\end{aligned}
$$

- Displacements $u_{d}^{y, j}$ at a point $(x, y)$ in an elastic layer $j$ when the load acts in the $y$ direction or in the fluid medium:

$$
\begin{aligned}
u_{x}^{y, j}= & E_{a}^{j} \sum_{n=-N}^{n=+N}\left(-\mathrm{i} k_{n} E_{b}^{a, j} A_{n}^{y, S_{j}}+\mathrm{i} k_{n} E_{c}^{a, j} B_{n}^{y, S_{j}}\right) E_{d} \\
& -E_{a}^{j} \sum_{n=-N}^{n=+N}\left(-\mathrm{i} k_{n} E_{b}^{b, j} D_{n}^{y, S_{j}}+\mathrm{i} k_{n} E_{c}^{b, j} E_{n}^{y, S_{j}}\right) E_{d}, \\
u_{y}^{y, j}= & E_{a}^{j} \sum_{n=-N}^{n=+N}\left(-\mathrm{i}{\nu_{n}^{j}}^{a, j} A_{b}^{y, S_{j}}-\frac{\mathrm{i} k_{n}^{2}}{\gamma_{n}^{j}} E_{c}^{a, j} B_{n}^{y, S_{j}}-\frac{\mathrm{i} k_{z}^{2}}{\gamma_{n}^{j}} E_{c}^{a, j} C_{n}^{y, S_{j}}\right) E_{d} \\
& +E_{a}^{j} \sum_{n=-N}^{n=+N}\left(-\mathrm{i} v_{n}^{j} E_{b}^{b, j} D_{n}^{y, S_{j}}-\frac{\mathrm{i} k_{n}^{2}}{\gamma_{n}^{j}} E_{c}^{b, j} E_{n}^{y, S_{j}}-\frac{\mathrm{i} k_{z}^{2}}{\gamma_{n}^{j}} E_{c}^{b, j} F_{n}^{y, S_{j}}\right) E_{d}, \\
u_{z}^{y, j}= & E_{a}^{j} \sum_{n=-N}^{n=+N}\left(-\mathrm{i} k_{z} E_{b}^{a, j} A_{n}^{y, S_{j}}+\mathrm{i} k_{z} E_{c}^{a, j} C_{n}^{y, S_{j}}\right) E_{d} \\
& -E_{a}^{j} \sum_{n=-N}^{n=+N}\left(-\mathrm{i} k_{z} E_{b}^{b, j} D_{n}^{y, S_{j}}+\mathrm{i} k_{z} E_{c}^{b, j} F_{n}^{y, S_{j}}\right) E_{d} .
\end{aligned}
$$

The proposed methodology was verified with a boundary element method (BEM) model that uses Green functions for an unbounded medium, requiring a large discretization of the solid-fluid interfaces; this has limitations because a large amount of damping has been introduced (not presented here).

Notice that in the present model the internal material loss is considered by using a complex Young's modulus and complex Lamé's constants. The Young's modulus is computed as $E=E_{r}(1+\mathrm{i} \eta)$, where $E_{r}$ corresponds to the classic modulus and $\eta$ is the loss factor. The complex Lamé's constants can be written in the same form as the Young's modulus. Note that in practical simulations the material damping is found to be much higher than that defined by the internal damping. 


\section{Validation of the analytical model}

In this section the analytical model is validated by comparing the responses against experimental results and simplified expressions. First the airborne sound insulation provided by single- and double-layered partitions is analyzed. Then the impact sound insulation provided by a single panel and a concrete screed floating system is discussed.

All simulations performed in this section consider elastic layers made of concrete $\left(c_{L}=3500 \mathrm{~m} / \mathrm{s} ; \quad c_{S}=2250 \mathrm{~m} / \mathrm{s} ; \quad \rho_{s}=2500 \mathrm{~kg} / \mathrm{m}^{3} ; \eta_{s}=4 \times 10^{-3}\right)$, glass $\left(c_{L}=5750 \mathrm{~m} / \mathrm{s} ;\right.$ $\left.c_{S}=3450 \mathrm{~m} / \mathrm{s} ; \rho_{s}=2500 \mathrm{~kg} / \mathrm{m}^{3} ; \eta_{s}=4 \times 10^{-3}\right)$ or steel $\left(c_{L}=6000 \mathrm{~m} / \mathrm{s} ; c_{S}=3200 \mathrm{~m} / \mathrm{s} ;\right.$ $\left.\rho_{s}=7850 \mathrm{~kg} / \mathrm{m}^{3} ; \eta_{s}=2 \times 10^{-2}\right)$. The layers divide a fluid medium which assumes the air properties $\left(\rho=1.2 \mathrm{~kg} / \mathrm{m}^{3}\right.$ and $\left.c=340 \mathrm{~m} / \mathrm{s}\right)$. The models are excited by point sources placed in the acoustic medium $\left(S_{f}\right)$ at position $(0.0 \mathrm{~m} ;-2.0 \mathrm{~m})$ or in the elastic medium $\left(S_{s}\right)$ acting in the $y$ direction at position $(0.0 \mathrm{~m} ; 0.0 \mathrm{~m})$ (see Fig. $\left.3 \mathrm{a}\right)$.

Several numerical simulations were performed to define the position of the receivers in the emitting and receiving media. It was found that the receivers should be placed in a line over large distances so as to capture features related to the propagation of guided waves, such as the coincidence effect. It was also concluded that good results were obtained for all simulations performed using the line of receivers displayed in Fig. 3a (placed $2.0 \mathrm{~m}$ apart in the acoustic media, on both sides of the panel). Similar results would be obtained for a larger line of receivers.

The responses presented in this paper are calculated in the frequency domain, using that set of receivers, for a frequency range of $[10.0 ; 8192.0 \mathrm{~Hz}]$ with a frequency increment of $2.0 \mathrm{~Hz}$.

\subsection{Airborne sound insulation}

The responses shown in this section refer to sound pressure level reduction. First the sound pressure is computed at the line of receivers displayed as in Fig. 3a, placed in the medium containing the source (to record the incident and reflected pressure field) and

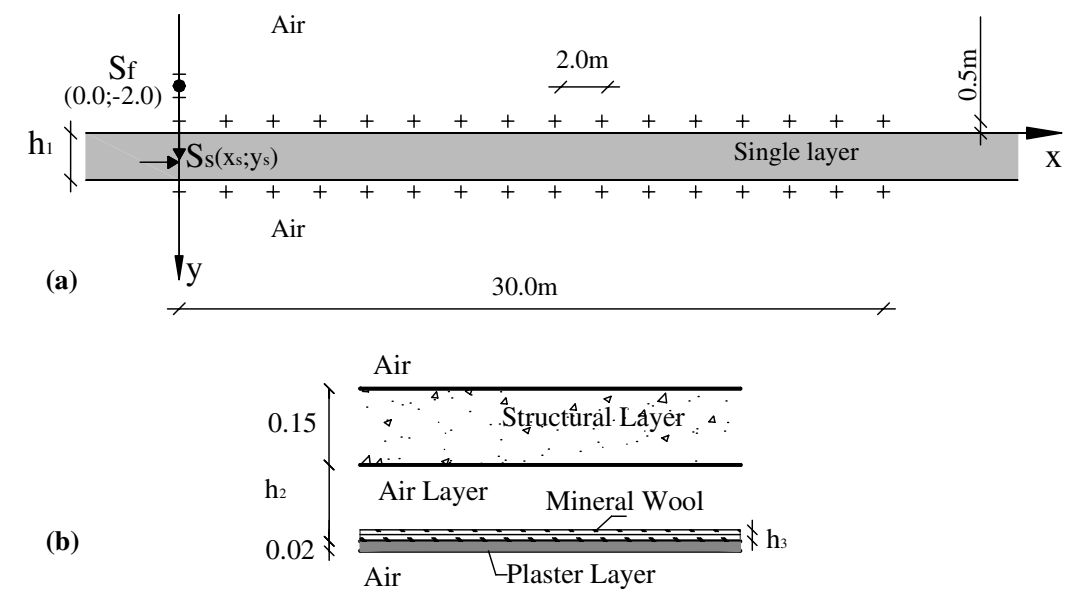

Fig. 3. Geometry of the simulations: (a) single structural layer with position of receivers and sources; (b) structural layer with a suspended plaster ceiling. 
in the receiving medium. Then the sound pressure level reduction is calculated by means of the difference between the ratio of the average of the sound pressure squared to the square of the reference sound pressure in the medium containing the source and in the receiving medium on a dB scale.

Fig. 4a shows the sound pressure level reduction provided by a single concrete layer $h_{1}=0.20 \mathrm{~m}$ thick (see Fig. 3a). As expected, the sound pressure level reduction increases as the frequency increases. The coincidence effect (labeled $\mathrm{fc}$ in the plot) is visible. This effect is associated with the propagation of guided waves along the structural layer, leading to increased movement of the panel and causing a drop in insulation. The propagation of these guided waves is often analyzed without taking into account the solid-fluid interaction. The mathematical development of these assumptions, not assuming the effects of the shear and rotary inertia, leads to the following simplified equation [14]:
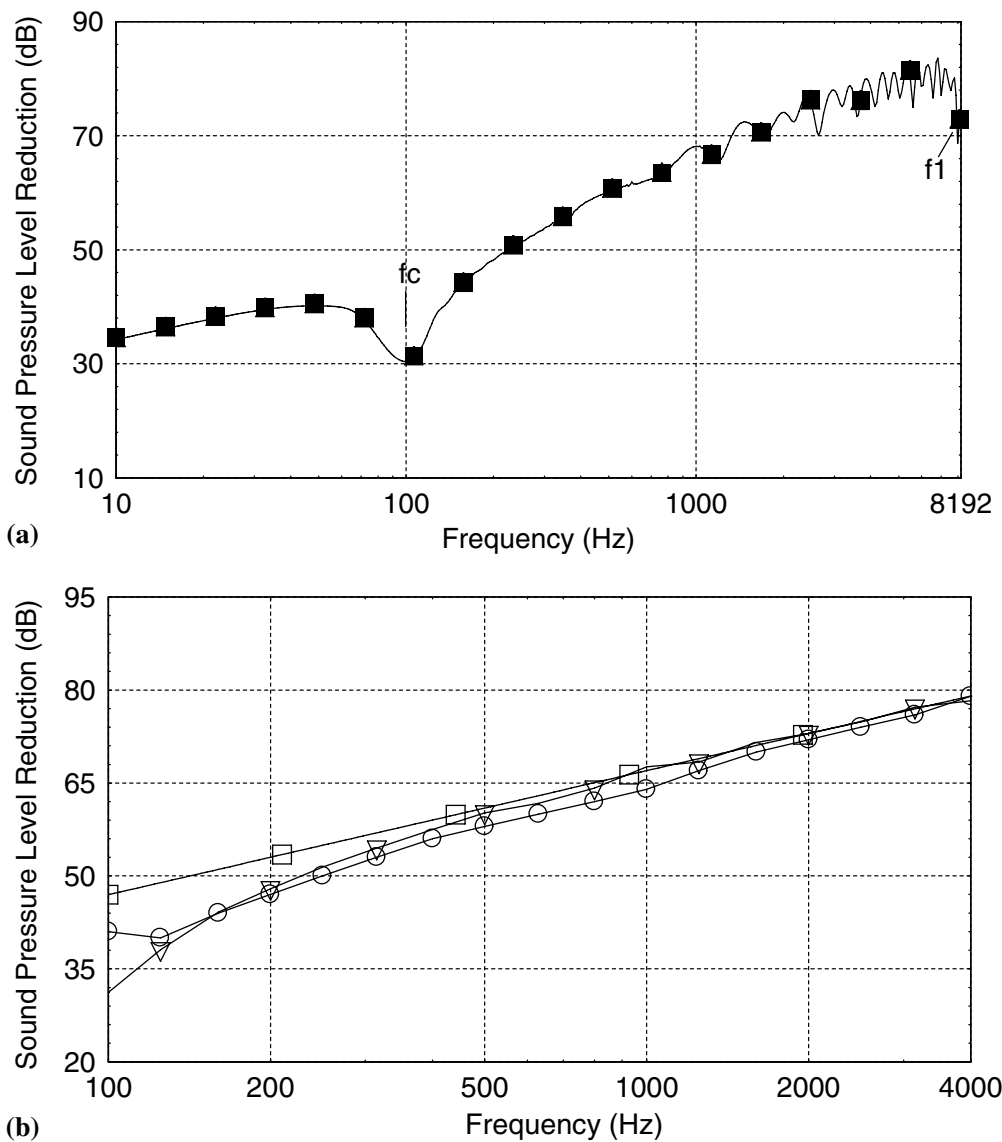

Fig. 4. Sound pressure level reduction provided by a single concrete structural layer $h_{1}=0.20 \mathrm{~m}$ thick using the: (a) analytical model ( $\square$ ); (b) analytical model $(\nabla)$, the mass law $(\square)$ and experimental result $(\bigcirc)$ in the $1 / 3$ octave band frequency domain. 


$$
\omega=\frac{c}{\sin \phi} \sqrt{\frac{\rho_{s} h_{1}}{D}},
$$

where $\rho_{s}$ is the density of the material $\left(\mathrm{kg} / \mathrm{m}^{3}\right), h_{1}$ is the thickness of the panel $(\mathrm{m})$; $D=h_{1}^{3} E /\left[12\left(1-v^{2}\right)\right]$ with $E$ and $v$ being the Young's modulus and the Poisson's ratio, respectively, and $\phi$ is the incidence angle of the sound relative to a direction perpendicular to the element. The critical frequency provided by the analytical response occurs at $100 \mathrm{~Hz}$, while expression (11) predicts $\mathrm{fc}=92.5 \mathrm{~Hz}$. Notice that this value is for plane waves traveling with an incidence of $\phi=90^{\circ}$, and the analytical response is calculated considering the incidence of a point load.

At higher frequencies, the curve is less smooth owing to the interaction between the incident and directly-reflected wave fields, which occurs when the difference in the travel paths is a multiple of the wavelength. Furthermore, at the end of the response a dip is being formed which is associated with resonances inside the panel (labeled $f_{1}$ in the plot) that is close to $f_{1}=\frac{c_{L}}{2 h_{1}}=\frac{3500}{2 \times 0.2}=8750 \mathrm{~Hz}$.

Fig. $4 \mathrm{~b}$ displays the sound pressure level reduction provided by the concrete structural panel $h_{1}=0.20 \mathrm{~m}$ thick, after performing an integration in the third octave band frequency. The plot also displays an experimental result (obtained from the database of the software Acoubat developed by CSTB [15]) and the curve provided by the mass law. Analysis of the curves allows the conclusion that the analytical result is in very good agreement with the experimental result throughout the frequency range, except for lower frequencies. This difference is due to the coincidence effect, which is not seen in the experimental result owing to the size of the specimen. Notice also that for lower frequencies the result provided by the mass law also differs from the experimental result. In fact the analytical model behaves better in the lower frequencies than the mass law.

Fig. 5 displays the sound pressure level reduction provided by a single concrete panel $h_{1}=0.04 \mathrm{~m}$ thick (Fig. 5a), a steel panel $h_{1}=0.01 \mathrm{~m}$ thick (Fig. 5b) and a single glass panel $h_{1}=0.004 \mathrm{~m}$ thick (Fig. 5c), using the analytical model and Lab results. The Lab tests were performed in accordance with ISO 140-1 [16] in the acoustic chambers of the Department of Civil Engineering's Constructions Lab in the University of Coimbra [17]. The acoustic chambers consist of an emitting room of $111 \mathrm{~m}^{3}$ and a receiving room $122 \mathrm{~m}^{3}$. The concrete and the glass panels tested in the chambers had an area of $1.25 \times 1.50 \mathrm{~m}^{2}$. The measurements were performed in accordance with ISO 140-3 [18] and the results are given in bands of $1 / 10$ octave. The curve provided by the mass law are included in the plots.

All the experimental results plotted in Fig. 5a-c are influenced by the stationary wave field within the two chambers that creates dips and fluctuations at low frequencies. These curves also show other dips related to the panel's transverse movement. These are influenced by the size of the panel and by the boundary conditions. Notice that the eigenmodes related to the panel's transverse movement and the stationary wave field created in the rooms facing the panel are not taken into account by the analytical model. Comparisons between the results provided by the mass law and the experimental results reveal differences between the curves. These differences become more pronounced as the frequency increases, when the coincidence frequency (labeled fc in the plots) starts to influence the responses.

A detailed analysis of Fig. 5a shows that the analytical response predicts a dip due to the coincidence effect which is not seen in the experimental result. In fact the laboratory 

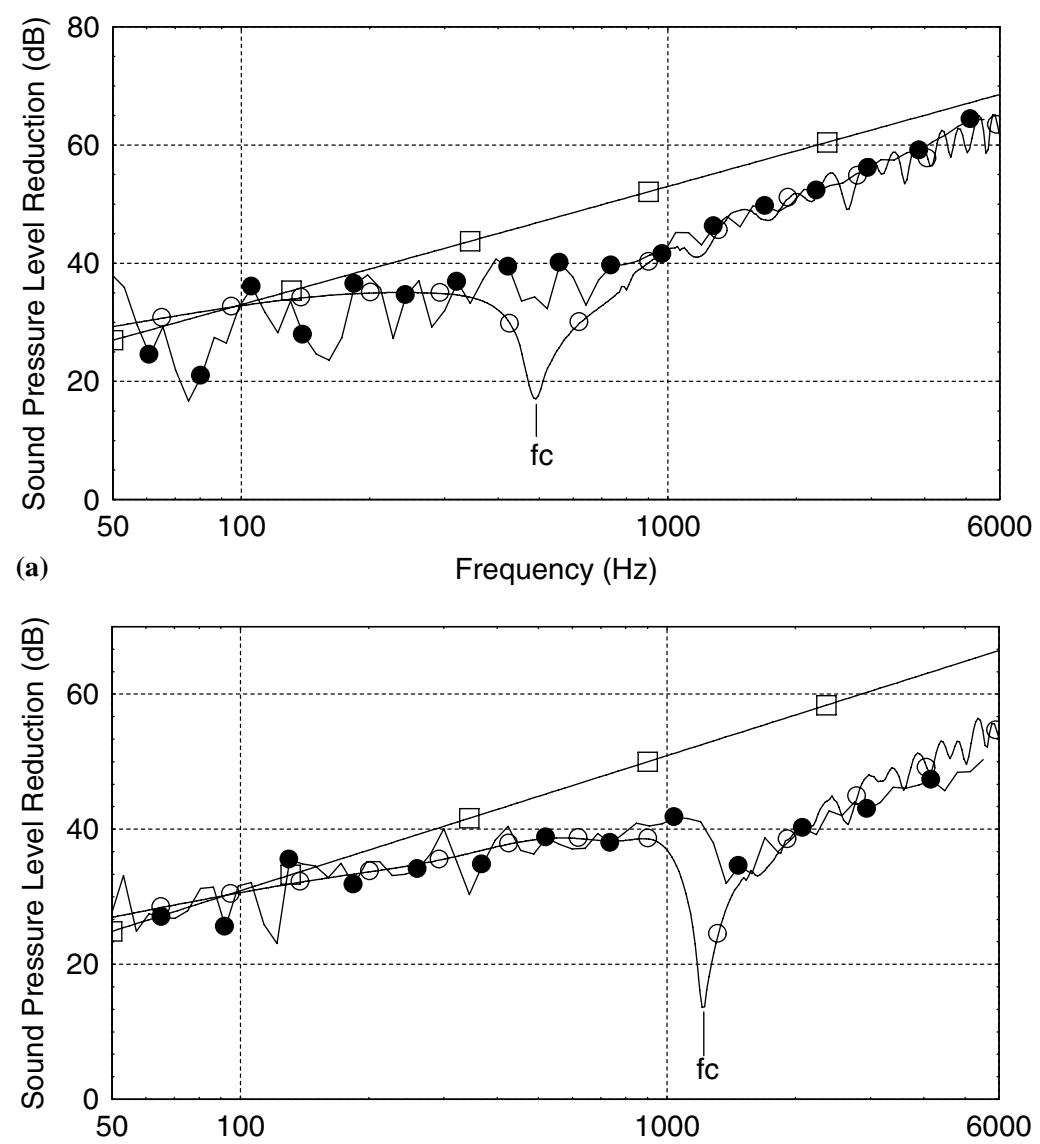

(b)

Frequency $(\mathrm{Hz})$

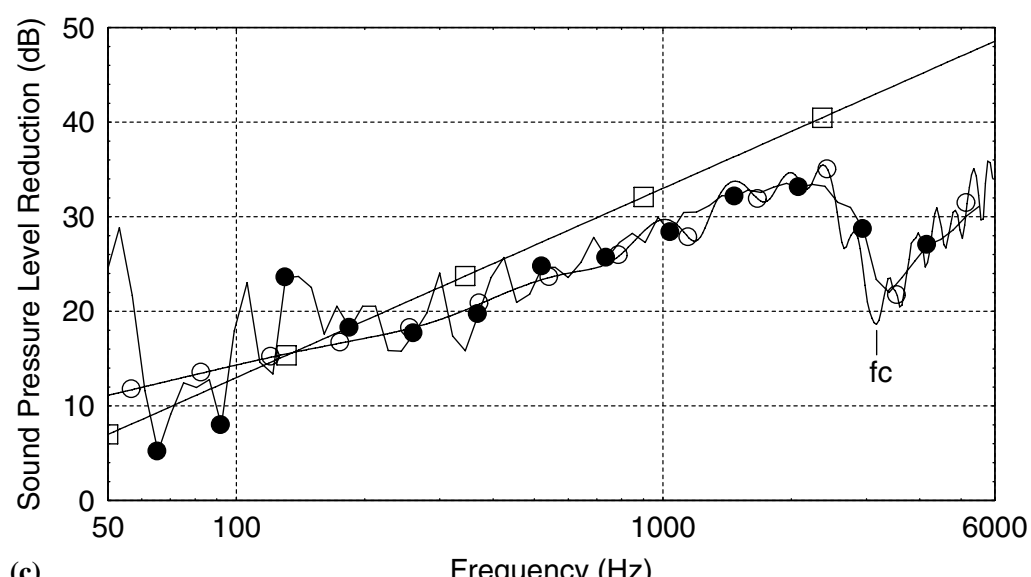

(c)

Frequency $(\mathrm{Hz})$

Fig. 5. Sound pressure level reduction using the analytical model $(\bigcirc)$ vs experimental results $(\bullet)$ vs the mass law ( $\square$ ) provided by a: (a) single concrete layer $h_{1}=0.04 \mathrm{~m}$ thick; (b) single steel layer $h_{1}=0.01 \mathrm{~m}$ thick; (c) single glass layer $h_{1}=0.004 \mathrm{~m}$ thick. 
test used a panel with an area of $1.25 \times 1.50 \mathrm{~m}^{2}$. The panel tested was not large enough for this phenomenon to be seen in the experimental response. At higher frequencies both curves present a very good agreement. Analysis of Fig. 5b allows similar conclusions to be drawn. When a glass panel is assumed (see Fig. 5c) the experimental curve exhibits the presence of the coincidence effect. Here the response provided by the analytical model shows an excellent agreement, even in the vicinity of the coincidence effect.

Fig. 6 exhibits the sound pressure level reduction provided by a double glazed panel $(4+4 \mathrm{~mm}$ sandwiching a $12 \mathrm{~mm}$ air layer). Fig. 6a plots the analytical response and the curve provided by the London-Beranek method [4] obtained when the panel is subjected to normally incident plane waves. Both curves predict a dip in the frequency associated with the mass-air-mass resonance (labeled $f_{\text {res }}$ in the plots) occurring at low frequencies.

The mass-spring-mass resonance frequency is computed here using the simplified expression:
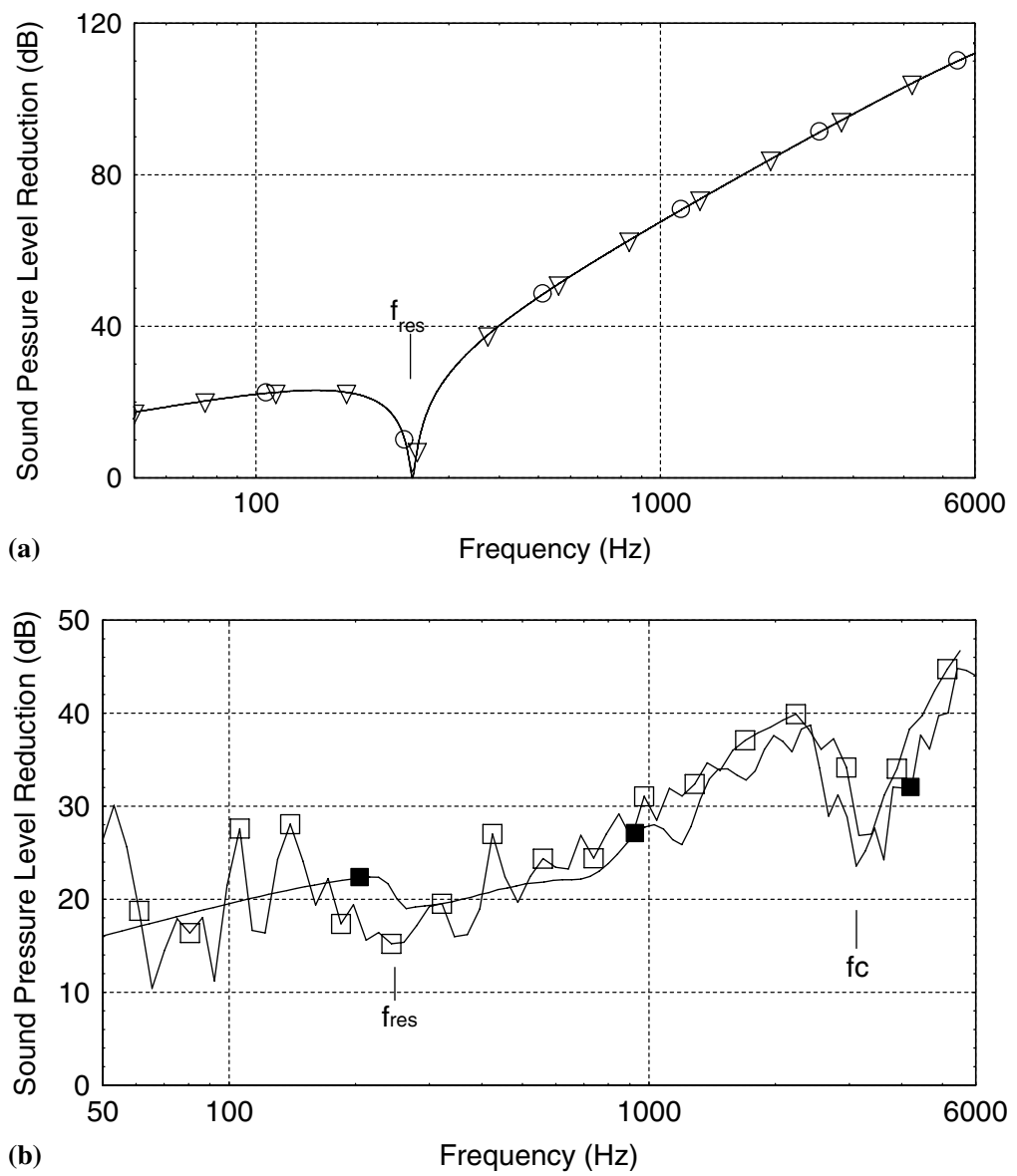

Fig. 6. Sound pressure level reduction provided by a double glazed panel $(4+12+4)$ : (a) responses provided by the analytical model $(\bigcirc)$ vs London-Beranek method $(\nabla)$ for the incidence of plane waves; (b) responses provided by the analytical model ( $\square$ ) vs experimental result $(\square)$ for the incidence of point pressure loads. 


$$
f_{\mathrm{res}}=\frac{1}{2 \pi} \sqrt{K\left(\frac{1}{m_{1}}+\frac{1}{m_{2}}\right)},
$$

where $K=\frac{c^{2} \rho_{f}}{h_{2}}$ with $c$ and $\rho_{f}$ being the dilatational wave velocity and the density of the air, respectively; $m_{1}$ and $m_{2}$ are the mass of each layer $\left(\mathrm{kg} / \mathrm{m}^{2}\right)$ and $h_{2}$ is the thickness of the air gap. The resonance of the mass-air-mass system predicted using expression (12) leads to $f_{\text {res }}=244 \mathrm{~Hz}$. This result is similar to that provided by the analytical model. Analysis of the figure reveals very good agreement between the analytical solution and the curve provided by London-Beranek method.

Fig. $6 \mathrm{~b}$ shows the analytical response provided by the double glazed panel when subjected to a point pressure load, and the experimental result obtained by testing a panel with an area of $1.25 \times 1.50 \mathrm{~m}^{2}$ [17]. All the results show dips associated with the massair-mass resonance and the coincidence effect. As before, the resonance effect inside the air layer is not visible as it occurs outside the frequency domain used in the analysis. Again the analytical result tends to show a good agreement with the experimental solution, except at low frequencies, owing to the fluctuations related to the stationary field generated in the emitting and receiving rooms of the chamber. Notice that the dip associated with the coincidence effect predicted by the analytical model is very similar with the experimental result.

The results presented above did not take into account the existence of flanking transmission through the side elements. In cases where this phenomenon may be relevant the sound pressure reduction will be lower than that found by the analytical model. In these situations, the contribution of flanking transmission can be calculated using the procedure described in EN 12354-1 [19].

\subsection{Impact sound pressure level}

In this section the impact sound pressure level results provided by the analytical model and by the experimental tests are discussed. The responses provided by the analytical model are obtained by calculating the ratio of the average of the sound pressure squared to the square of the reference sound pressure recorded at the receivers placed in the receiving medium, as shown in Fig. 3a.

Fig. 7 displays the results provided by a single concrete layer $h_{1}=0.20 \mathrm{~m}$ thick. Fig. $7 \mathrm{a}$ shows the response provided by the analytical model simulating a theoretical impact from a standard tapping machine. The results show that the sound level increases in the vicinity of both the coincidence effect and the resonances inside the elastic layer (labeled as $f_{1}$ in the plot).

Fig. 7b plots the analytical and experimental results provided by the concrete layer under the action of a standard tapping machine (obtained from the software Acoubat developed by CSTB [15]). In order to compare our results with the experimental curve, the amplitude of the impact load is defined in the frequency domain so as to model the response provided by a standard tapping machine. The frequency spectrum of the impact load is obtained from the approach followed by Cremer [8]. According to this author the impact provided by the tapping machine (rate of the hammer strikes $-10 \mathrm{~Hz}$ ) on a high impedance structure, in the frequency domain, exhibits a constant amplitude of $8.859 \mathrm{~N}$. The results are shown in the $1 / 3$ octave frequency bands. The two curves in 

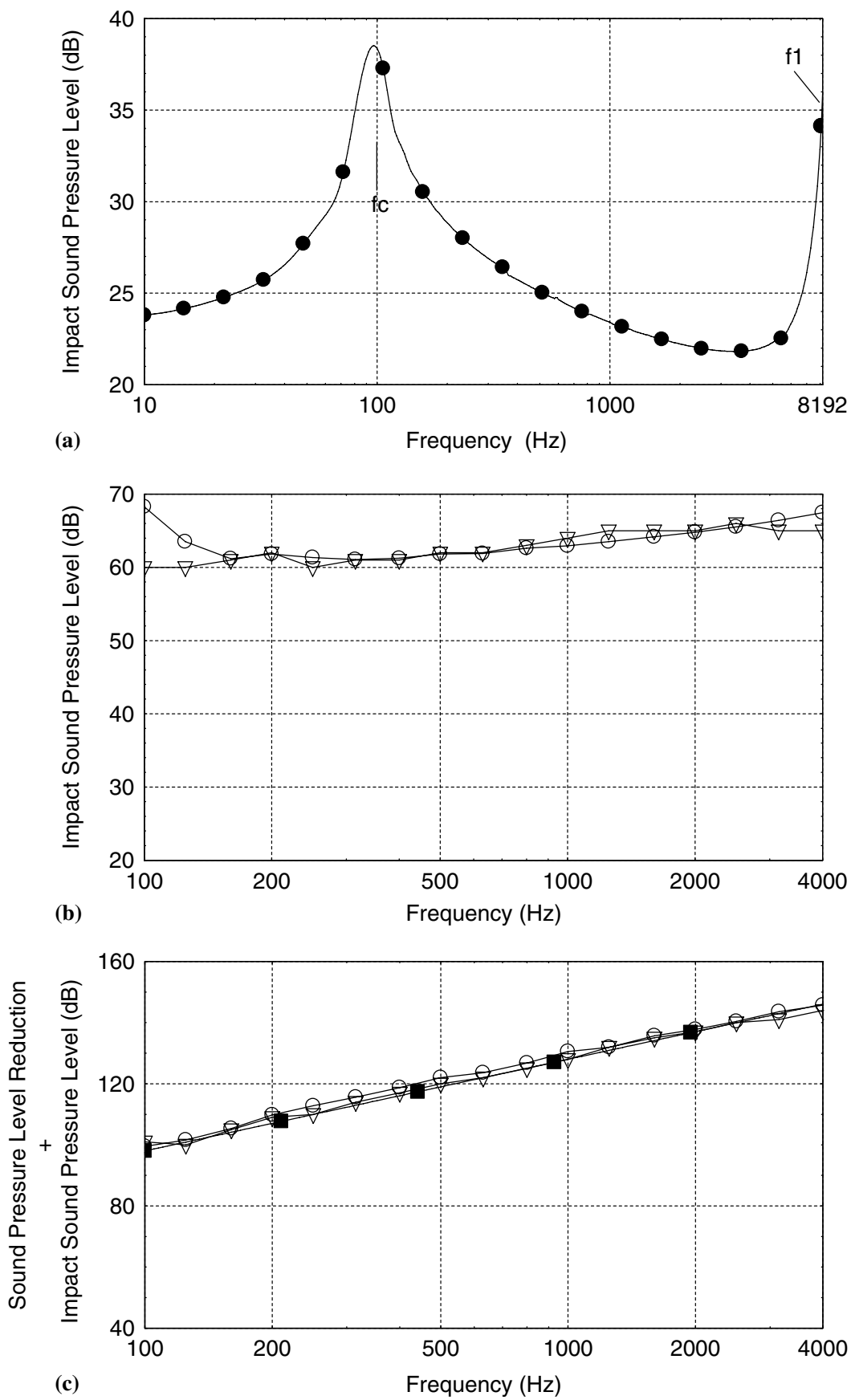

Fig. 7. Frequency domain responses provided by a single concrete structure $h_{1}=0.20 \mathrm{~m}$ thick: (a) impact sound pressure level provided by the analytical model $(\mathbf{O})$; (b) impact sound pressure level provided by the analytical model $(O)$ vs experimental results $(\nabla)$ in the $1 / 3$ octave band frequency domain; (c) sum of sound pressure level reduction and impact sound pressure level in the $1 / 3$ octave band frequency domain $(\bigcirc$ analytical result; $\nabla$ experimental result; $\mathbf{D e c k l}$ and Rathe expression). 
the plot exhibit similar behavior, except at the lower frequencies. In fact the experimental response provided by the concrete floors is not influenced by the coincidence effect that is predicted by the analytical model.

The results provided by the analytical model are also compared with the simplified expression achieved by Heckl et al. [10], modified for $1 / 3$ octave frequency bands,

$$
L_{n}+R=38+30 \log \left(f_{m}\right),
$$

where $f_{m}$ is the third octave band center frequency in $\mathrm{Hz} ; R$ is the sound transmission loss of an element, and $L_{n}$ is the impact sound pressure level, defined as the sound level measured in the receiving room when a standard tapping machine is operating. This expression assumes that the coincidence frequency is low and the surface is hard and has high input impedance. According to the authors, this relation does not hold if there is a hole in the element, which allows the waves produced by the pressure source and the impact sound waves to travel along different path, or if the flanking transmission through the side walls is dominant in relation to that occurring through the structural layer.

The sum of airborne and impact sound insulation is computed for the single concrete layer and integrations in the $1 / 3$ octave band frequency are performed. Responses provided by the analytical model, experimental results and expression (13) are plotted in Fig. 7c. The results show that the two curves exhibit similar behavior. The good agreement that is found between curves is related to the fact that the proposed model is based on the assumption that the panels are of infinite extent, meaning that the results do not account for flanking transmission.

Fig. 8 displays the analytical and experimental results provided by a floating concrete screed layer. The model consists of a concrete layer $0.04 \mathrm{~m}$ thick resting on a resilient quilt laid over a structural concrete layer, $0.14 \mathrm{~m}$ thick. The resilient quilt is marketed by Imperalum under the name Impersom [20], and the experimental result was obtained from a test performed in the acoustic chambers of the National Laboratory of Civil Engineering,

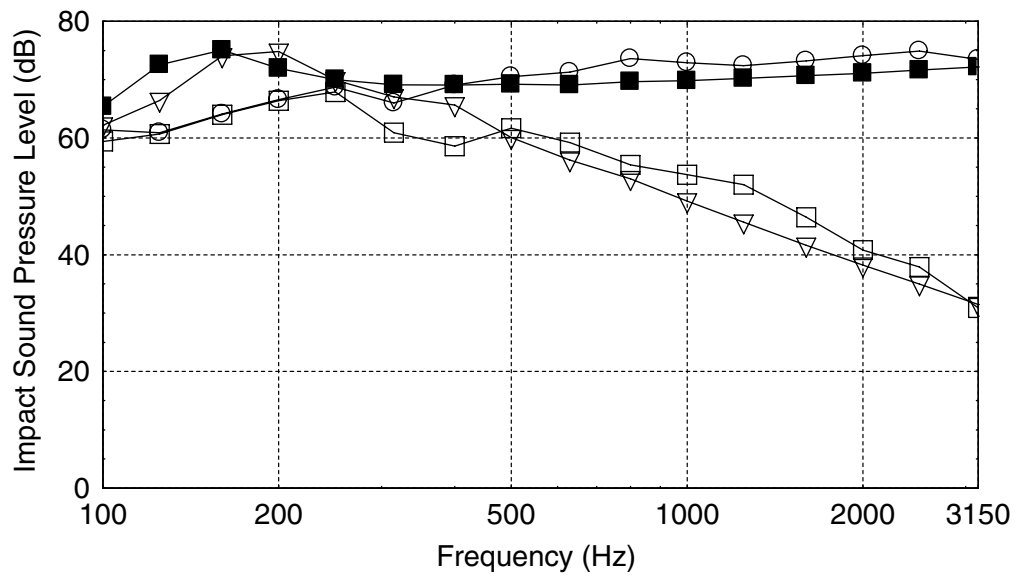

Fig. 8. Impact sound pressure level provided by a single concrete layer $h_{1}=0.14 \mathrm{~m}$ thick and by a concrete-screed floating layer, in the $1 / 3$ octave band frequency domain $(\bigcirc$ experimental result of the single concrete layer; $\square$ experimental result of the concrete-screed floating layer; $\mathbf{a n a l y t i c a l}$ result of the single concrete layer; $\nabla$ analytical result of the concrete-screed floating layer). 
according to ISO 140-1 [16]. The specimen has an area of $10 \mathrm{~m}^{2}$ and the test was performed following the procedure of ISO 140-8 [21]. The plot shows the impact sound level provided by both the single concrete layer and by the concrete screed floating layer. Analysis of the responses shows that at low frequencies the analytical curves are influenced by the coincidence effect, and so differences are found between these and the experimental results. However at medium and high frequencies the curves exhibit good agreement.

When there is relevant flanking transmission the analytical results can be corrected using the techniques described in EN 12354:2 [22].

\section{Applicability of the analytical model}

This section illustrates the applicability of the proposed model with a selection of results provided by the analytical model for a single panel with varying materials and thicknesses and a concrete structural layer with a suspended ceiling (see Fig. 3).

All simulations performed consider layers, made of concrete (properties given in the previous section), ceramic material $\left(c_{L}=2200 \mathrm{~m} / \mathrm{s} ; c_{S}=1350 \mathrm{~m} / \mathrm{s} ; \rho_{s}=1400 \mathrm{~kg} / \mathrm{m}^{3} ; \eta_{s}=\right.$ $\left.1.5 \times 10^{-2}\right)$ or plasterboard $\left(c_{L}=2200 \mathrm{~m} / \mathrm{s} ; \quad c_{S}=1250 \mathrm{~m} / \mathrm{s} ; \quad \rho_{s}=820 \mathrm{~kg} / \mathrm{m}^{3} ; \quad \eta_{s}=3 \times\right.$ $\left.10^{-2}\right)$. The load in the acoustic medium $\left(S_{f}\right)$ is located, as before, at position $(0.0 \mathrm{~m}$; $-2.0 \mathrm{~m}$ ), whereas in the elastic medium it can either act in the $y$ (vertical load) or in the $x$ (horizontal load) directions, at different positions.

\subsection{Acoustic behavior of a single panel}

Fig. 9a shows the sound pressure level reduction provided by a single structural layer made either of ceramic or concrete and assuming a thickness of $h_{1}=0.10 \mathrm{~m}$ or $h_{1}=0.20 \mathrm{~m}$. As expected, analysis of the curves reveals that insulation increases when mass increases. When the stiffness of the element increases the critical frequency appears at lower frequencies and the dip in insulation in the vicinity of this frequency is lower.

Fig. $9 \mathrm{~b}$ and $\mathrm{c}$ displays the impact sound insulation when either vertical or horizontal unit loads, $S_{s}$ excite a concrete single panel with thickness $h_{1}=0.20 \mathrm{~m}$. Notice that the loads may act not perpendicularly to the plane of a structure, determined by the existence of vertical and horizontal components. In the presence of certain mechanical equipment the horizontal component may even be dominant. Thus, when the source is placed inside the elastic medium the influence of the depth and the direction of the load on the impact sound insulation are studied by considering four different loads at depths $y_{s}=0.0 \mathrm{~m}$, $y_{s}=0.05 \mathrm{~m}, y_{s}=0.10 \mathrm{~m}$ and $y_{s}=0.15 \mathrm{~m}$. For all the four loads, the horizontal position is kept at $x_{s}=0.0 \mathrm{~m}$. When the load acts vertically (see Fig. 9b), the results show that the sound level increases in the vicinity of both the coincidence effect and the resonances inside the elastic layer. Comparing the curves obtained for different source depths, we see that the source position does not seem to influence the responses. In fact, the only major difference among the responses is found at high frequencies when the source is placed at $y_{s}=0.10 \mathrm{~m}$ (the middle of the layer), when some vibration modes of the layer are not excited.

When a horizontally-acting impact load excites the panel (see Fig. 9c), the increase in sound level in the vicinity of the coincidence effect occurs for all the source depths considered, except for $y_{s}=0.10 \mathrm{~m}$. When the source is placed at the surface, the resulting sound level is higher than for the other source positions. In fact, the responses show that the 

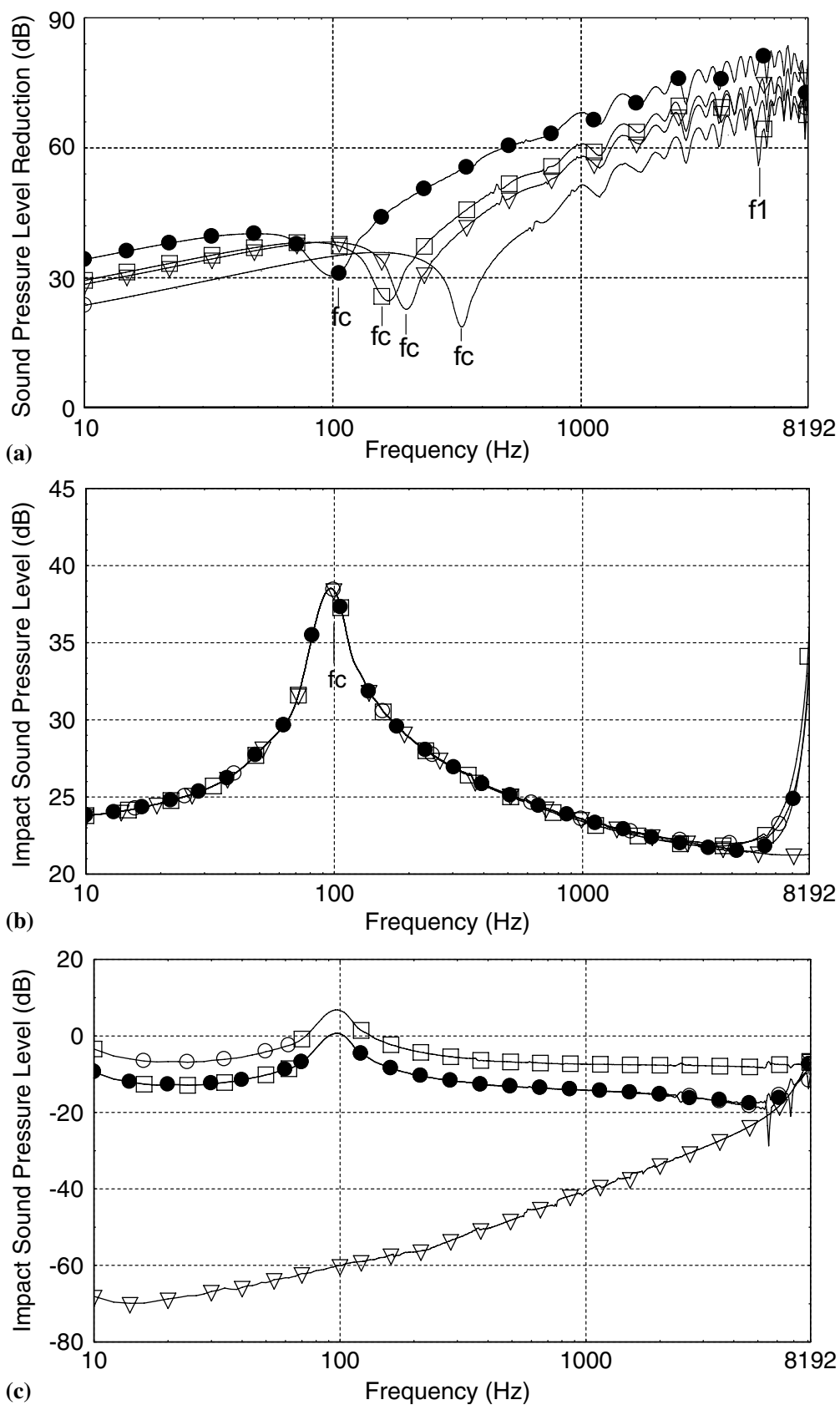

Fig. 9. Frequency domain responses provided by a single structure: (a) sound pressure level reduction provided by a single layer made of concrete with $h_{1}=0.10 \mathrm{~m}(\nabla)$ and $h_{1}=0.20 \mathrm{~m}(\bullet)$ and of ceramic with $h_{1}=0.10 \mathrm{~m}(\bigcirc)$

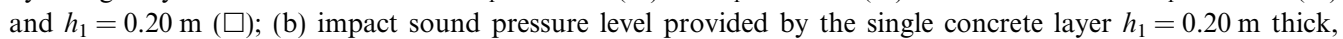
when the source, placed at different depths $y_{s}$, acts in the $y$ direction; (c) impact sound pressure level provided by the single concrete layer $h_{1}=0.20 \mathrm{~m}$ thick, when the source, placed at different source depths $y_{s}$, acts in the $x$ direction $\left(\bigcirc y_{s}=0.00 \mathrm{~m} ; \square y_{s}=0.05 \mathrm{~m} ; \nabla y_{s}=0.10 \mathrm{~m} ; \bullet y_{s}=0.15 \mathrm{~m}\right)$. 
sound level in the receiving space is highly influenced by the source depth. When the depth is $y_{s}=0.10 \mathrm{~m}$ the influence of the propagating guided waves does not seem to be important and the impact insulation appears to be much lower than that obtained for the other positions. Moreover, sound level increases as frequency increases. Comparison with the responses shown in Fig. 9b indicates that the contribution to impact sound insulation of the source acting horizontally is lower than when the load acts vertically. When the load acts horizontally more energy travels along the panel and less is radiated into the receiving medium.

\subsection{Acoustic behavior of a concrete layer with a suspended ceiling}

The last example analyses the dynamic responses of a suspended ceiling when porous material partially or totally fills the air gap. The geometry is built using a $0.15 \mathrm{~m}$ concrete structural layer with a suspended ceiling built with an air gap of $h_{2}=0.15 \mathrm{~m}$ and a plasterboard layer $0.02 \mathrm{~m}$ thick (see Fig. 3b). Simulations with mineral wool material with a density $\rho=50.00 \mathrm{~kg} / \mathrm{m}^{3}$ inside the air layer are also considered, and results when this material is taken to have thickness $h_{3}=0.00 \mathrm{~m}$ (empty air gap), $h_{3}=0.15 \mathrm{~m}$ (filled air gap) and $h_{3}=0.02 \mathrm{~m}$ (filling lying against the plaster layer) are discussed. The absorbing material is modeled by ascribing a complex air density, which depends on the flow resistivity, on the porosity of the material and on a structure factor [5].

Simulations are performed using mineral wool with a density $\rho=50.00 \mathrm{~kg} / \mathrm{m}^{3}$ (which corresponds to a flow resistivity of $\left.1000 \mathrm{~kg} / \mathrm{m}^{3} / \mathrm{s}[5]\right)$.

Fig. 10 displays both the sound pressure level reduction (Fig. 10a) and the impact sound pressure level (Fig. 10b) when there is no mineral wool layer present $\left(h_{3}=0.00 \mathrm{~m}\right.$ - empty air gap), and for $h_{3}=0.02 \mathrm{~m}$ and $h_{3}=0.15 \mathrm{~m}$ (fully-filled) thick mineral wool layers. Responses obtained for a $0.15 \mathrm{~m}$ thick single concrete structural layer are used as a reference. As before the source acts in the acoustic medium at $(0.0 \mathrm{~m} ;-2.0 \mathrm{~m})$ and in the elastic medium in the $y$ direction at $(0.0 \mathrm{~m} ; 0.0 \mathrm{~m})$.

All results show a decrease in insulation in the vicinity of the critical frequency associated with the propagation of guided waves along the concrete structural layer (labeled $\mathrm{fc}$ in the plots). The frequency associated with the mass-air-mass resonance (labeled $f_{\text {res }}$ in the plots) occurs at low frequencies and produces a fall in insulation. The resonance of the mass-air-mass system predicted using expression (12) leads to $f_{\text {res }}=38.1 \mathrm{~Hz}$. This result is similar to that provided by the analytical model. The propagation of guided waves along the plaster layer does not seem to be important. The resonances excited inside the air gap create a sharp dip in insulation at specific frequencies, labeled in the plot as $f_{1}, f_{2}, \ldots, f_{6}$. These frequencies can be predicted by $f_{m}=\mathrm{cm} /\left(2 h_{2}\right), m=1,2, \ldots$, where $h_{2}$ refers to the thickness of the air gap. According to the analytical model, they lie at higher frequencies than those predicted by this expression. It should be noted that the simplified expression assumes that the source only emits plane waves that travel perpendicularly to the panel. When the mineral wool quilt is placed inside the air layer, the resonance effect inside the air gap and the coincidence effect are both attenuated, and insulation improves in the vicinity of these frequencies. Notice that the insulation provided by the concrete structural layer with a suspended ceiling layer is much better than that provided by a single $0.15 \mathrm{~m}$ concrete structural layer for the range of frequencies analyzed. 


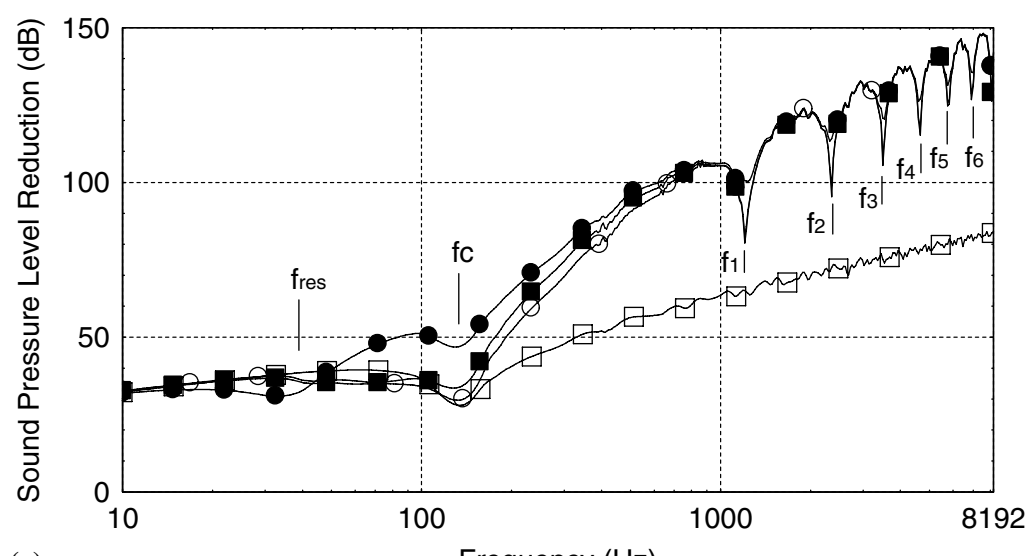

(a)

Frequency $(\mathrm{Hz})$

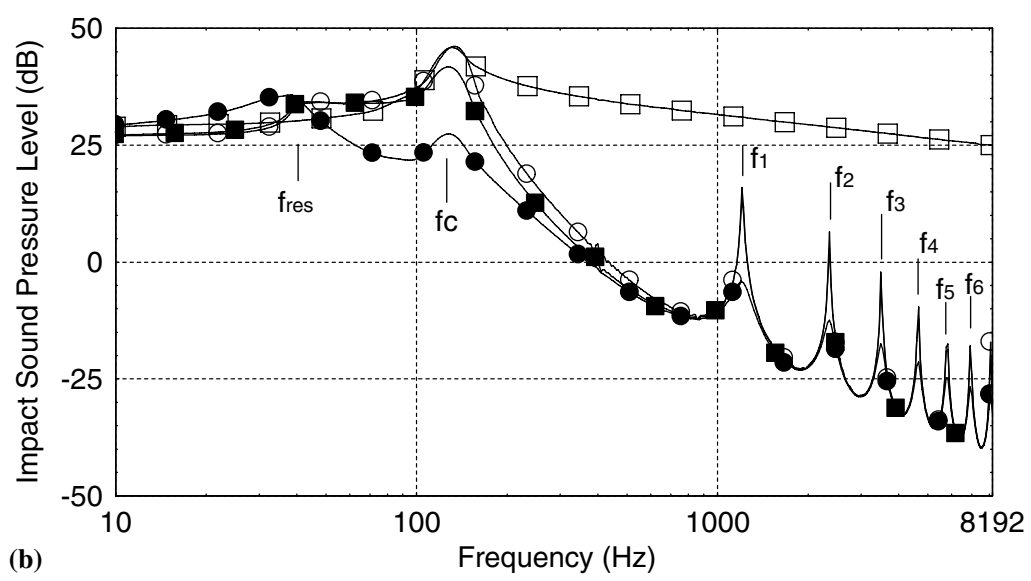

Fig. 10. Frequency domain responses provided by a suspended plaster ceiling lined with mineral wool $h_{3}=0.00 \mathrm{~m}(\bigcirc), h_{3}=0.02 \mathrm{~m}(\mathbf{\square})$ and $h_{3}=0.15 \mathrm{~m}(\mathbf{O})$ and by a single concrete layer $h_{1}=0.15 \mathrm{~m}(\square):$ (a) sound pressure level reduction; (b) impact sound pressure level when the source acts in the $y$ direction.

\section{Conclusions}

An analytical model, based on wave propagation theory for layered medium, has been developed to predict the airborne and impact sound insulation provided by single and multilayer systems of infinite extent, neglecting sound bridges and flanking transmission. Responses are calculated in the frequency domain using point loads.

The model was validated by comparing the responses with experimental results and with simplified formulas. This comparison was performed for airborne sound insulation provided by single panels of varying thickness and double panels. A good agreement between experimental and numerical results was found in the generality of the analyzed partitions. Differences were found: at lower frequencies due to the stationary wave field occurring inside the chambers and to the panels' transverse modes; in the frequencies defining the coincidence effect of thicker panels, which were related to the size of the tested 
panels. It was found that the results provided by the analytical model show a better agreement with the experimental results than those provided by the mass law. Impact sound insulation was also calculated for a single panel and a floating layer system and validation was performed with experimental results. Again the analytical responses are quite similar to the experimental ones. The major differences are located at the lower frequencies in the vicinity of the coincidence effect.

The applicability of the analytical solutions to the prediction of the acoustic behavior of a single structural layer and a suspended ceiling configuration was then discussed. It was shown that the proposed model is able to capture all the physical acoustic phenomena involved in the prediction of the acoustic behavior provided by single and multilayer systems of infinite extent, such as: the mass-air-mass resonance phenomena, the coincidence effect associated with the propagation of guided waves of the individual panels, the resonances excited inside the air gap and the effect of having the air layer filled with mineral wool.

\section{Appendix 1}

This appendix lists the individual systems of equations that are established for each layer.

- Within the fluid layer:

$$
\left[\begin{array}{c}
\sigma^{a, j} \\
u_{y}^{a, j} \\
\sigma^{b, j} \\
u_{y}^{b, j}
\end{array}\right]=m_{k, l}^{F_{j}}(k=1, \ldots, 4 ; l=1,2)\left[\begin{array}{c}
G_{n}^{F_{j}} \\
H_{n}^{F_{j}}
\end{array}\right],
$$

where

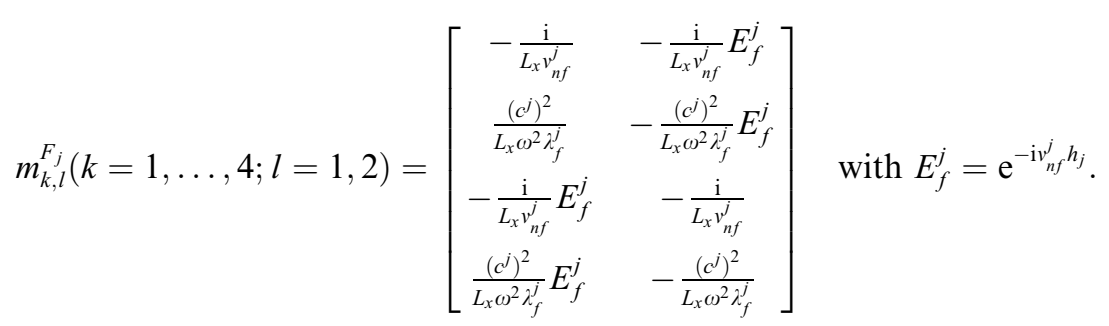

- Within the elastic layer when the load acts along the $x$ direction:

$$
\left[\begin{array}{c}
\sigma_{y x}^{x, a, j} \\
\sigma_{y z}^{x, a, j} \\
\sigma_{y y}^{x, a, j} \\
u_{x}^{x, a, j} \\
u_{y}^{x, a, j} \\
u_{z}^{x, a, j}
\end{array}\right]=E_{a}^{j} m_{(k=1, \ldots, 6 ; l=1, \ldots, 6)}^{x, S_{j}}\left[\begin{array}{c}
A_{n}^{x, S_{j}} \\
B_{n}^{x, S_{j}} \\
C_{n}^{x, S_{j}} \\
D_{n}^{x, S_{j}} \\
E_{n}^{x, S_{j}} \\
F_{n}^{x, S_{j}}
\end{array}\right]
$$




$$
\left[\begin{array}{c}
\sigma_{y x}^{x, b, j} \\
\sigma_{y z}^{x, b, j} \\
\sigma_{y y}^{x, b, j} \\
u_{x}^{x, b, j} \\
u_{y}^{x, b, j} \\
u_{z}^{x, b, j}
\end{array}\right]=E_{a}^{j} m_{(k=7, \ldots, 12 ; l=1, \ldots, 6)}^{x, S_{j}}\left[\begin{array}{c}
A_{n}^{x, S_{j}} \\
B_{n}^{x, S_{j}} \\
C_{n}^{x, S_{j}} \\
D_{n}^{x, S_{j}} \\
E_{n}^{x, S_{j}} \\
F_{n}^{x, S_{j}}
\end{array}\right],
$$

where

$$
\begin{aligned}
& m_{(k=1, \ldots, 6, l=1, \ldots, 6)}^{x, S_{j}} \\
& =\left[\begin{array}{cccccc}
-2 \mu^{j} k_{n}^{2} & -\mu^{j} k_{z}^{2} & \mu^{j}\left(k_{n}^{2}-\left(\gamma_{n}^{j}\right)^{2}\right) & 2 \mu^{j} k_{n}^{2} E_{b h}^{j} & \mu^{j} k_{z}^{2} E_{c h}^{j} & -\mu^{j}\left(k_{n}^{2}-\left(\gamma_{n}^{j}\right)^{2}\right) E_{c h}^{j} \\
-2 \mu^{j} & \mu^{j} & \mu^{j} & 2 \mu^{j} E_{b h}^{j} & -\mu^{j} E_{c h}^{j} & -\mu^{j} E_{c h}^{j} \\
-2 \frac{\mu^{j} k_{n}}{v_{n}^{j}}\left(v_{z n}^{2}+\frac{\left(k_{s}^{j}\right)^{2}}{2}\right) & 0 & 2 \mu^{j} k_{n} \gamma_{n}^{j} & -2 \frac{\mu^{j} k_{n}}{v_{n}^{j}}\left(v_{z n}^{2}+\frac{\left(k_{s}^{j}\right)^{2}}{2}\right) E_{b h}^{j} & 0 & 2 \mu^{j} k_{n} \gamma_{n}^{j} E_{c h}^{j} \\
-\mathrm{i} \frac{k_{n}^{2}}{v_{n}^{j}} & -\mathrm{i} \frac{k_{z}^{2}}{\gamma_{n}^{j}} & -\mathrm{i} \gamma_{n}^{j} & -\mathrm{i} \frac{k_{n}^{2}}{v_{n}^{j}} E_{b h}^{j} & -\mathrm{i} \frac{k_{z}^{2}}{\gamma_{n}^{j}} E_{c h}^{j} & -\mathrm{i} \gamma_{n}^{j} E_{c h}^{j} \\
-\mathrm{i} k_{n} & 0 & \mathrm{i} k_{n} & \mathrm{i} k_{n} E_{b h}^{j} & 0 & -\mathrm{i} k_{n} E_{c h}^{j} \\
-\frac{\mathrm{i}}{v_{n}^{j}} & \frac{\mathrm{i}}{\gamma_{n}^{j}} & 0 & -\frac{\mathrm{i}}{v_{n}^{j}} E_{b h}^{j} & \frac{\mathrm{i}}{\gamma_{n}^{j}} E_{c h}^{j} & 0
\end{array}\right], \\
& m_{(k=7, \ldots, 12, l=1, \ldots, 6)}^{x, S_{j}} \\
& =\left[\begin{array}{cccccc}
-2 \mu^{j} k_{n}^{2} E_{b h}^{j} & -\mu^{j} k_{z}^{2} E_{c h}^{j} & \mu^{j}\left(k_{n}^{2}-\left(\gamma_{n}^{j}\right)^{2}\right) E_{c h}^{j} & 2 \mu^{j} k_{n}^{2} & \mu^{j} k_{z}^{2} & -\mu^{j}\left(k_{n}^{2}-\left(\gamma_{n}^{j}\right)^{2}\right) \\
-2 \mu^{j} E_{b h}^{j} & \mu^{j} E_{c h}^{j} & \mu^{j} E_{c h}^{j} & 2 \mu^{j} & -\mu^{j} & -\mu^{j} \\
-2 \frac{\mu^{j} k_{n}}{v_{n}^{j}}\left(v_{z n}^{2}+\frac{\left(k_{s}^{j}\right)^{2}}{2}\right) E_{b h}^{j} & 0 & 2 \mu^{j} k_{n} \gamma_{n}^{j} E_{c h}^{j} & -2 \frac{\mu^{j} k_{n}}{v_{n}^{\prime}}\left(v_{z n}^{2}+\frac{\left(\frac{\left(s_{s}^{j}\right)^{2}}{2}\right)}{0}\right. & 0 & 2 \mu^{j} k_{n} \gamma_{n}^{j} \\
-\mathrm{i} \frac{k_{n}^{2}}{v_{n}^{j}} E_{b h}^{j} & -\mathrm{i} \frac{k_{z}^{2}}{\gamma_{n}^{\prime}} E_{c h}^{j} & -\mathrm{i} \gamma_{n}^{j} E_{c h}^{j} & -\mathrm{i} \frac{k_{n}^{2}}{v_{n}^{j}} & -\mathrm{i} \frac{k_{z}^{2}}{\gamma_{n}^{j}} & -\mathrm{i} \gamma_{n}^{j} \\
-\mathrm{i} k_{n} E_{b h}^{j} & 0 & \mathrm{i} k_{n} E_{c h}^{j} & \mathrm{i} k_{n} & 0 & -\mathrm{i} k_{n} \\
-\frac{\mathrm{i}}{v_{n}^{j}} E_{b h}^{j} & \frac{\mathrm{i}}{\gamma_{n}^{j}} E_{c h}^{j} & 0 & -\frac{\mathrm{i}}{v_{n}^{j}} & \frac{\mathrm{i}}{\gamma_{n}^{j}} & 0
\end{array}\right]
\end{aligned}
$$

with $E_{b h}^{j}=\mathrm{e}^{-\mathrm{i} v_{n}^{j} h_{j}} ; E_{c h}^{j}=\mathrm{e}^{-\mathrm{i} \gamma_{n}^{j^{h}} h_{j}} ; v_{z n}=\sqrt{-k_{z}^{2}-k_{n}^{2}}$.

- Within the elastic layer when the load acts along the $y$ direction or in a fluid layer:

$$
\begin{aligned}
& {\left[\begin{array}{c}
\sigma_{y x}^{y, a, j} \\
\sigma_{y z}^{y, a, j} \\
\sigma_{y y}^{y, a, j} \\
u_{x}^{y, a, j} \\
u_{y}^{y, a, j} \\
u_{z}^{y, a, j}
\end{array}\right]=E_{a}^{j} m_{(k=1, \ldots, 6 ; l=1, \ldots, 6)}^{y, S_{j}}\left[\begin{array}{c}
A_{n}^{y, S_{j}} \\
B_{n}^{y, S_{j}} \\
C_{n}^{y, S_{j}} \\
D_{n}^{y, S_{j}} \\
E_{n}^{y, S_{j}} \\
F_{n}^{y, S_{j}}
\end{array}\right],} \\
& {\left[\begin{array}{c}
\sigma_{y x}^{y, b, j} \\
\sigma_{y z}^{y, b, j} \\
\sigma_{y y}^{y, b, j} \\
u_{x}^{y, b, j} \\
u_{y}^{y, b, j} \\
u_{z}^{y, b, j}
\end{array}\right]=E_{a}^{j} m_{(k=7, \ldots, 12 ; l=1, \ldots, 6)}^{y, S_{j}}\left[\begin{array}{c}
A_{n}^{y, S_{j}} \\
B_{n}^{y, S_{j}} \\
C_{n}^{y, S_{j}} \\
D_{n}^{y, S_{j}} \\
E_{n}^{y, S_{j}} \\
F_{n}^{y, S_{j}}
\end{array}\right],}
\end{aligned}
$$


where

$$
\begin{aligned}
& m_{(k=1, \ldots, 6, l=1, \ldots, 6)}^{y, S_{j}} \\
& =\left[\begin{array}{cccccc}
-2 \mu^{j} v_{n}^{j} & \mu^{j}\left(-\frac{k_{n}^{2}}{\gamma_{n}^{j}}+\gamma_{n}^{j}\right) & -\mu^{j} \frac{k_{z}^{2}}{\gamma_{n}^{j}} & -2 \mu^{j} v_{n}^{j} E_{b h}^{j} & \mu^{j}\left(-\frac{k_{n}^{2}}{\gamma_{n}^{j}}+\gamma_{n}^{j}\right) E_{c h}^{j} & -\mu^{j} \frac{k_{z}^{2}}{\gamma_{n}^{j}} E_{c h}^{j} \\
-2 \mu^{j} v_{n}^{j} & -\mu^{j} \frac{k_{n}^{2}}{\gamma_{n}^{n}} & \mu^{j}\left(-\frac{k_{z}^{2}}{\gamma_{n}^{\prime}}+\gamma_{n}^{j}\right) & -2 \mu^{j} v_{n}^{j} E_{b h}^{j} & -\mu^{j} \frac{k_{n}^{2}}{\gamma_{n}^{\prime}} E_{c h}^{j} & \mu^{j}\left(-\frac{k_{z}^{2}}{\gamma_{n}^{n}}+\gamma_{n}^{j}\right) E_{c h}^{j} \\
-2 \mu^{j}\left(v_{z n}^{2}+\frac{\left(k_{s}^{j}\right)^{2}}{2}\right) & -2 \mu^{j} k_{n}^{2} & -2 \mu^{j} k_{z}^{2} & 2 \mu^{j}\left(v_{z n}^{2}+\frac{\left(k_{s}^{j}\right)^{2}}{2}\right) E_{b h}^{j} & 2 \mu^{j} k_{n}^{2} E_{c h}^{j} & 2 \mu^{j} k_{z}^{2} E_{c h}^{j} \\
-\mathrm{i} & \mathrm{i} & 0 & \mathrm{i} E_{b h}^{j} & -\mathrm{i} E_{c h}^{j} & 0 \\
-\mathrm{i} v_{n}^{j} & -\mathrm{i} \frac{k_{n}^{2}}{\gamma_{n}^{\prime}} & -\mathrm{i} \frac{k_{z}^{2}}{\gamma_{n}^{\prime}} & -\mathrm{i} v_{n}^{j} E_{b h}^{j} & -\mathrm{i} \frac{k_{n}^{2}}{\gamma_{n}^{\prime}} E_{c h}^{j} & -\mathrm{i} \frac{k_{z}^{2}}{\gamma_{n}^{2}} E_{c h}^{j} \\
-\mathrm{i} & 0 & \mathrm{i} & \mathrm{i} E_{b h}^{j} & 0 & -\mathrm{i} E_{c h}^{j}
\end{array}\right], \\
& m_{(k=7, \ldots, 12, l=1, \ldots, 6)}^{y, S_{j}} \\
& =\left[\begin{array}{cccccc}
-2 \mu^{j} v_{n}^{j} E_{b h}^{j} & \mu^{j}\left(-\frac{k_{n}^{2}}{\gamma_{n}^{j}}+\gamma_{n}^{j}\right) E_{c h}^{j} & -\mu^{j} \frac{k_{z}^{2}}{\gamma_{n}^{2}} E_{c h}^{j} & -2 \mu^{j} v_{n}^{j} & \mu^{j}\left(-\frac{k_{n}^{2}}{\gamma_{n}^{j}}+\gamma_{n}^{j}\right) & -\mu^{j} \frac{k_{z}^{2}}{\gamma_{n}^{j}} \\
-2 \mu^{j} v_{n}^{j} E_{b h}^{j} & -\mu^{j} \frac{k_{n}^{2}}{\gamma_{n}^{j}} E_{c h}^{j} & \mu^{j}\left(-\frac{k_{z}^{2}}{\gamma_{n}^{j}}+\gamma_{n}^{j}\right) E_{c h}^{j} & -2 \mu^{j} v_{n}^{j} & -\mu^{j} \frac{k_{n}^{2}}{\gamma_{n}^{j}} & \mu^{j}\left(-\frac{k_{z}^{2}}{\gamma_{n}^{j}}+\gamma_{n}^{j}\right) \\
-2 \mu^{j}\left(v_{z n}^{2}+\frac{\left(k_{s}^{j}\right)^{2}}{2}\right) E_{b h}^{j} & -2 \mu^{j} k_{n}^{2} E_{c h}^{j} & -2 \mu^{j} k_{z}^{2} E_{c h}^{j} & 2 \mu^{j}\left(v_{z n}^{2}+\frac{\left(k_{s}^{j}\right)^{2}}{2}\right) & 2 \mu^{j} k_{n}^{2} & 2 \mu^{j} k_{z}^{2} \\
-\mathrm{i} E_{b h}^{j} & \mathrm{i} E_{c h}^{j} & 0 & \mathrm{i} & -\mathrm{i} & 0 \\
-\mathrm{i} v_{n}^{j} E_{b h}^{j} & -\mathrm{i} \frac{k_{n}^{2}}{\gamma_{n}^{2}} E_{c h}^{j} & -\mathrm{i} \frac{k_{z}^{2}}{\gamma_{n}^{j}} E_{c h}^{j} & -\mathrm{i} v_{n}^{j} & -\mathrm{i} \frac{k_{n}^{2}}{\gamma_{n}^{j}} & -\mathrm{i} \frac{k_{z}^{2}}{\gamma_{n}^{j}} \\
-\mathrm{i} E_{b h}^{j} & 0 & \mathrm{i} E_{c h}^{j} & \mathrm{i} & 0 & -\mathrm{i}
\end{array}\right]
\end{aligned}
$$

The incident field within the layer where the load is applied is given as:

- Load acting in the fluid layer:

$$
\operatorname{inc}_{(k=1, \ldots, 4)}^{f, j}=\left[\begin{array}{c}
\sigma^{\mathrm{inc}, a, j} \\
u_{y}^{\mathrm{inc}, a, j} \\
\sigma^{\mathrm{inc}, b, j} \\
u_{y}^{\mathrm{inc}, b, j}
\end{array}\right]=\left[\begin{array}{c}
-\frac{\mathrm{i}}{L_{x} v_{n f}^{j}} \mathrm{e}^{-\mathrm{i} v_{n f}^{j}\left|y^{a, j}-y_{0}\right|} \\
-\frac{\left(\alpha_{f}^{j}\right)^{2}}{L_{x} \omega^{2} \lambda_{f}^{j}} \mathrm{e}^{-\mathrm{i} v_{n f}^{j}\left|y^{a, j}-y_{0}\right|} \\
-\frac{\mathrm{i}}{L_{x} v_{n f}^{j}} \mathrm{e}^{-\mathrm{i} v_{n f}^{j}\left|y^{b, j}-y_{0}\right|} \\
\frac{\left(c^{j}\right)^{2}}{L_{x} \omega^{2} \lambda_{f}^{j}} \mathrm{e}^{-\mathrm{i} v_{n f}^{j}\left|y^{b, j}-y_{0}\right|}
\end{array}\right]
$$

- Load acting in the $x$ direction is placed in the elastic layer:

$$
\operatorname{inc}_{(k=1, \ldots, 6)}^{x, j}=\left[\begin{array}{c}
\sigma_{y x}^{x, \text { inc }, a, j} \\
\sigma_{y z}^{x, \text { inc }, a, j} \\
\sigma_{y y}^{x, \text { inc }, a, j} \\
u_{x}^{x, \text { inc }, a, j} \\
u_{y}^{x, \text { inc }, a, i} \\
u_{z}^{x, \text { inc }, a, j}
\end{array}\right]=E_{a}^{j}\left[\begin{array}{c}
2 \mu^{j} k_{n}^{2} E_{b}^{s, a, j}+\mu^{j} k_{z}^{2} E_{c}^{s, a, j}-\mu^{j}\left(k_{n}^{2}-\left(\gamma_{n}^{j}\right)^{2}\right) E_{c}^{s, a, j} \\
2 \mu^{j} E_{b}^{s, a, j}-\mu^{j} E_{c}^{s, a, j}-\mu^{j} E_{c}^{s, a, j} \\
-2 \frac{\mu^{j} k_{n}}{v_{n}^{j}}\left(v_{z n}^{2}+\frac{\left(k_{s}^{j}\right)^{2}}{2}\right) E_{b}^{s, a, j}+2 \frac{\mu^{j} k_{n}}{v_{n}^{j}}\left(v_{z n}^{2}+\frac{\left(k_{s}^{j}\right)^{2}}{2}\right) E_{c}^{s, a, j} \\
-\mathrm{i} \frac{k_{n}^{2}}{v_{n}^{j}} E_{b}^{s, a, j}-\mathrm{i} \frac{k_{z}^{2}}{\gamma_{n}^{i}} E_{c}^{s, a, j}-\mathrm{i} \gamma_{n}^{j} E_{c}^{s, a, j} \\
\mathrm{i} k_{n} E_{b}^{s, a, j}-\mathrm{i} k_{n} E_{c}^{s, a, j} \\
-\frac{\mathrm{i}}{v_{n}^{j}} E_{b}^{s, a, j}+\frac{\mathrm{i}}{\gamma_{n}^{i}} E_{c}^{s, a, j}
\end{array}\right],
$$




$$
\operatorname{inc}_{(k=7, \ldots, 12)}^{x, j}=\left[\begin{array}{c}
\sigma_{y x}^{x, \text { inc }, b, j} \\
\sigma_{y z}^{x, \text { inc }, b, j} \\
\sigma_{y y}^{x, \text { inc }, b, j} \\
u_{x}^{x, \text { inc }, b, j} \\
u_{y}^{x, \text { inc }, b, j} \\
u_{z}^{x, \text { inc }, b, j}
\end{array}\right]=E_{a}^{j}\left[\begin{array}{c}
-2 \mu^{j} k_{n}^{2} E_{b}^{s, b, j}-\mu^{j} k_{z}^{2} E_{c}^{s, b, j}+\mu^{j}\left(k_{n}^{2}-\left(\gamma_{n}^{j}\right)^{2}\right) E_{c}^{s, b, j} \\
-2 \mu^{j} E_{b}^{s, b, j}+\mu^{j} E_{c}^{s, b, j}+\mu^{j} E_{c}^{s, b, j} \\
-2 \frac{\mu^{j} k_{n}}{v_{n}^{j}}\left(v_{z n}^{2}+\frac{\left(k_{s}^{j}\right)^{2}}{2}\right) E_{b}^{s, b, j}+2 \frac{\mu^{j} k_{n}}{v_{n}^{j}}\left(v_{z n}^{2}+\frac{\left(k_{s}^{j}\right)^{2}}{2}\right) E_{c}^{s, b, j} \\
-\mathrm{i} \frac{k_{n}^{2}}{v_{n}^{j}} E_{b}^{s, b, j}-\mathrm{i} \frac{k_{z}^{2}}{\gamma_{n}^{j}} E_{c}^{s, b, j}-\mathrm{i} \gamma_{n}^{j} E_{c}^{s, b, j} \\
-\mathrm{i} k_{n} E_{b}^{s, b, j}+\mathrm{i} k_{n} E_{c}^{s, b, j} \\
-\frac{\mathrm{i}}{v_{n}^{j}} E_{b}^{s, b, j}+\frac{\mathrm{i}}{\gamma_{n}^{\prime}} E_{c}^{s, b, j}
\end{array}\right],
$$

where $E_{b}^{s, a, j}=\mathrm{e}^{-\mathrm{i} v_{n}^{j}\left|y^{a, j}-y_{0}\right|} ; E_{b}^{s, b, j}=\mathrm{e}^{-\mathrm{i} v_{n}^{j}\left|y^{b, j}-y_{0}\right|} ; E_{c}^{s, a, j}=\mathrm{e}^{-\mathrm{i} \gamma_{n}^{j}\left|y^{a, j}-y_{0}\right|} ; E_{c}^{s, b, j}=\mathrm{e}^{-\mathrm{i} \gamma_{n}^{j}\left|y^{b, j}-y_{0}\right|}$.

- Load acting in the $y$ direction is placed in the elastic layer:

$$
\begin{aligned}
& \operatorname{inc}_{(k=1, \ldots, 6)}^{y, j}=\left[\begin{array}{c}
\sigma_{y x}^{y, \text { inc }, a, j} \\
\sigma_{y z}^{y, \text { inc }, a, j} \\
\sigma_{y y}^{y, \text { inc }, a, j} \\
u_{x}^{y, \text { inc }, a, j} \\
u_{y}^{y, \text { inc }, a, j} \\
u_{z}^{y, \text { inc }, a, j}
\end{array}\right]=E_{a}^{j}\left[\begin{array}{c}
-2 \mu^{j} v_{n}^{j} E_{b}^{s, a, j}+\mu^{j}\left(-\frac{k_{n}^{2}}{\gamma_{n}^{j}}+\gamma_{n}^{j}\right) E_{c}^{s, a, j}-\mu^{j} \frac{k_{z}^{2}}{\gamma_{n}^{j}} E_{c}^{s, a, j} \\
-2 \mu^{j} v_{n}^{j} E_{b}^{s, a, j}-\mu^{j} \frac{k_{n}^{2}}{\gamma_{n}^{j}} E_{c}^{s, a, j}+\mu^{j}\left(-\frac{k_{n}^{2}}{\gamma_{n}^{j}}+\gamma_{n}^{j}\right) E_{c}^{s, a, j} \\
\left.2 \mu_{z n}^{2}+\frac{\left(k_{s}^{j}\right)^{2}}{2}\right) E_{b}^{s, a, j}+2 \mu^{j} k_{n}^{2} E_{c}^{s, a, j}+2 \mu^{j} k_{z}^{2} E_{c}^{s, a, j} \\
\mathrm{i} E_{b}^{s, a, j}-\mathrm{i} E_{c}^{s, a, j} \\
-\mathrm{i} v_{n}^{j} E_{b}^{s, a, j}-\mathrm{i} \frac{k_{n}^{2}}{\gamma_{n}^{j}} E_{c}^{s, a, j}-\mathrm{i} \frac{k_{z}^{2}}{\gamma_{n}^{j}} E_{c}^{s, a, j} \\
\mathrm{i} E_{b}^{s, a, j}-\mathrm{i} E_{c}^{s, a, j}
\end{array}\right], \\
& \operatorname{inc}_{(k=7, \ldots, 12)}^{y, j}\left[\begin{array}{c}
\sigma_{y x}^{y, \text { inc }, b, j} \\
\sigma_{y z}^{y, \text { inc }, b, j} \\
\sigma_{y y}^{y, \text { inc }, b, j} \\
u_{x}^{y, \text { inc }, b, j} \\
u_{y}^{y, \text { inc }, b, j} \\
u_{z}^{y, \text { inc }, b, j}
\end{array}\right]=E_{a}^{j}\left[\begin{array}{c}
-2 \mu^{j} v_{n}^{j} E_{b}^{s, b, j}+\mu^{j}\left(-\frac{k_{n}^{2}}{\gamma_{n}^{j}}+\gamma_{n}^{j}\right) E_{c}^{s, b, j}-\mu^{j} \frac{k_{z}^{2}}{\gamma_{n}^{j}} E_{c}^{s, b, j} \\
-2 \mu^{j} v_{n}^{j} E_{b}^{s, b, j}-\mu^{j} \frac{k_{n}^{2}}{\gamma_{n}^{j}} E_{c}^{s, b, j}+\mu^{j}\left(-\frac{k_{n}^{2}}{\gamma_{n}^{j}}+\gamma_{n}^{j}\right) E_{c}^{s, b, j} \\
-2 \mu^{j}\left(v_{z n}^{2}+\frac{\left(k_{s}^{j}\right)^{2}}{2}\right) E_{b}^{s, b, j}-2 \mu^{j} k_{n}^{2} E_{c}^{s, b, j}-2 \mu^{j} k_{z}^{2} E_{c}^{s, b, j} \\
-\mathrm{i} E_{b}^{s, b, j}+\mathrm{i} E_{c}^{s, b, j} \\
-\mathrm{i} v_{n}^{j} E_{b}^{s, b, j}-\mathrm{i} \frac{k_{n}^{2}}{\gamma_{n}^{j}} E_{c}^{s, b, j}-\mathrm{i} \frac{k_{z}^{2}}{\gamma_{n}^{j}} E_{c}^{s, b, j} \\
-\mathrm{i} E_{b}^{s, b, j}+\mathrm{i} E_{c}^{s, b, j}
\end{array}\right] .
\end{aligned}
$$

\section{Appendix 2}

The multilayer system defined in Fig. 1 , built with a set of elastic $\left(S_{j}\right.$ with $\left.j=1, \ldots, n\right)$ and fluid layers $\left(F_{j}\right.$ with $\left.j=1, \ldots, n\right)$, is used to illustrate how the final system of equations is built, assuming that a pressure load placed in the Fluid 1 excites it. The final system of equations is obtained by combining the individual systems of equations, defined in Appendix 1, and prescribing the boundary conditions listed in Table A2.1.

The final system of equations takes the form:

$$
A X=B,
$$


Table A2.1

Boundary conditions at the interfaces

\begin{tabular}{|c|c|}
\hline Interfaces & Boundary conditions \\
\hline$\overline{a, S_{1}}$ & $\begin{array}{l}\sigma_{y x}^{a, S_{1}}=0 ; \sigma_{y z}^{a, S_{1}}=0 ; \sigma^{b, F_{1}}-\sigma_{y y}^{a, S_{1}}=\sigma^{i n c, b, F_{1}} \\
u_{y}^{b, F_{1}}-u_{y}^{a, S_{1}}=u_{y}^{i n c, b, F_{1}}\end{array}$ \\
\hline$b, S_{1}$ or $a, S_{2}$ & $\begin{array}{l}\sigma_{y x}^{b, S_{1}}=\sigma_{y x}^{a, S_{2}} ; \sigma_{y z}^{b, S_{1}}=\sigma_{y z}^{a, S_{2}} ; \sigma_{y y}^{b, S_{1}}=\sigma_{y y}^{a, S_{2}} \\
u_{x}^{b, S_{1}}=u_{x}^{a, S_{2}} ; u_{y}^{b, S_{1}}=u_{y}^{a, S_{2}} ; u_{z}^{b, S_{1}}=u_{z}^{a, S_{2}}\end{array}$ \\
\hline$b, S_{2}$ & $\begin{array}{l}\sigma_{y x}^{b, S_{2}}=\sigma_{y x}^{a, S_{3}} ; \sigma_{y z}^{b, S_{2}}=\sigma_{y z}^{a, S_{3}} ; \sigma_{y y}^{b, S_{2}}=\sigma_{y y}^{a, S_{3}} \\
u_{x}^{b, S_{2}}=u_{x}^{a, S_{3}} ; u_{y}^{b, S_{2}}=u_{y}^{a, S_{3}} ; u_{z}^{b, S_{2}}=u_{z}^{a, S_{3}}\end{array}$ \\
\hline$\cdots$ & $\cdots$ \\
\hline$a, F_{n-1}$ & $\begin{array}{l}\sigma_{x, x_{n-2}}^{b, S_{n}}=0 ; \sigma_{y z}^{b, S_{n-2}}=0 ; \sigma_{y y}^{b, S_{n-2}}=\sigma^{a, F_{n-1}} \\
u_{y}^{b, S_{n-2}}=u_{y}^{a, F_{n-1}}\end{array}$ \\
\hline$b, F_{n-1}$ or $a, S_{n}$ & $\begin{array}{l}\sigma_{y x}^{a, S_{n}}=0 ; \sigma_{y z}^{a, S_{n}}=0 ; \sigma^{b, F_{n-1}}=\sigma_{y y}^{a, S_{n}} \\
u_{y}^{b, F_{n-1}}=u_{y}^{a, S_{n}}\end{array}$ \\
\hline$b, S_{n}$ & $\begin{array}{l}\sigma_{x x}^{b, S_{n}}=0 ; \sigma_{y z}^{b, S_{n}}=0 ; \sigma_{y y}^{b, S_{n}}=\sigma^{a, F_{2}} \\
u_{y}^{b, S_{n}}=u_{y}^{a, F_{2}}\end{array}$ \\
\hline
\end{tabular}

\section{where}

\begin{tabular}{|c|c|c|c|c|c|c|}
\hline 0 & $-m_{(k=1 ; l=1, \ldots, 6)}^{S_{1}}$ & 0 & $\cdots$ & 0 & 0 & 0 \\
\hline 0 & $-m_{(k=2 ; l=1, \ldots, 6)}^{S_{1}}$ & 0 & $\ldots$ & 0 & 0 & 0 \\
\hline$m_{(k=3 ; l=2)}^{F_{1}}$ & $-m_{(k=3 ; l=1, \ldots, 6)}^{S_{1}}$ & 0 & $\cdots$ & 0 & 0 & 0 \\
\hline$m_{(k=4 ; l=2)}^{F_{1}}$ & $-m_{(k=5 ; l=1, \ldots, 6)}^{S_{1}}$ & 0 & $\cdots$ & 0 & 0 & 0 \\
\hline 0 & $-m_{(k=7, \ldots, 12 ; l=1, \ldots, 6)}^{S_{1}}$ & $m_{(k=1, \ldots, 6 ; l=1, \ldots, 6)}^{S_{2}}$ & $\ldots$ & 0 & 0 & 0 \\
\hline 0 & 0 & $m_{(k=7, \ldots, 12 ; l=1, \ldots, 6)}^{S 2}$ & $\cdots$ & 0 & 0 & 0 \\
\hline$\ldots$ & $\ldots$ & $\ldots$ & $\ldots$ & $\ldots$ & $\ldots$ & $\ldots$ \\
\hline 0 & 0 & 0 & $\cdots \quad m_{(k=7 ; l=1, \ldots, 6)}^{S_{n-2}}$ & 0 & 0 & 0 \\
\hline 0 & 0 & 0 & $\cdots \quad m_{(k=8 ; l=1, \ldots, 6)}^{S_{n-2}}$ & 0 & 0 & 0 \\
\hline 0 & 0 & 0 & $\cdots \quad m_{(k=9 ; l=1, \ldots, 6)}^{S_{n-2}}$ & $-m_{(k=1 ; l=1,2)}^{F_{n-1}}$ & 0 & 0 \\
\hline 0 & 0 & 0 & $\cdots m_{(k=11 ; l=1, \ldots, 6)}^{S_{n-2}}$ & $-m_{(k=2 ; l=1,2)}^{F_{n-1}}$ & 0 & 0 \\
\hline 0 & 0 & 0 & $\ldots$ & 0 & $-m_{(k=1: l=1, \ldots, 6)}^{S_{n}}$ & 0 \\
\hline 0 & 0 & 0 & $\cdots$ & 0 & $-m_{(k=2 ; l=1, \ldots, 6)}^{S_{S}}$ & 0 \\
\hline 0 & 0 & 0 & $\ldots$ & $m_{(k=3 ; l=1,2)}^{F_{n-1}}$ & $-m_{(k=3 ; l=1, \ldots, 6)}^{S_{n}}$ & 0 \\
\hline 0 & 0 & 0 & $\ldots$ & $m_{(k=4 ; l=1,2)}^{F_{n-1}}$ & $-m_{(k=5 ; l=1, \ldots, 6)}^{S_{n}}$ & 0 \\
\hline 0 & 0 & 0 & $\ldots$ & 0 & $m_{(k=7 ; l=1, \ldots, 6)}^{S_{n}}$ & 0 \\
\hline 0 & 0 & 0 & $\ldots$ & 0 & $m_{(k=8 ; l=1, \ldots, 6)}^{S_{n}}$ & 0 \\
\hline 0 & 0 & 0 & $\ldots$ & 0 & $m_{(k=9 ; l=1, \ldots, 6)}^{S_{n}}$ & $-m_{(k=1 ; l=1)}^{F_{2}}$ \\
\hline 0 & 0 & 0 & $\ldots$ & 0 & $m_{(k=11 ; l=1, \ldots, 6)}^{S_{n}}$ & $-m_{(k=2 ; l=}^{F_{2}}$ \\
\hline
\end{tabular}

and

$$
X=\left[\begin{array}{lllllllllllllllll}
H_{n}^{F_{1}} & A_{n}^{S_{1}} & \cdots & F_{n}^{S_{1}} & A_{n}^{S_{2}} & \cdots & F_{n}^{S_{2}} & \cdots & A_{n}^{S_{n-2}} & \cdots & F_{n}^{S_{n-2}} & G_{n}^{F_{n-1}} & H_{n}^{F_{n-1}} & A_{n}^{S_{n}} & \cdots & F_{n}^{S_{n}} & G_{n}^{F_{2}}
\end{array}\right]^{\mathrm{T}} .
$$


If the source is placed in Fluid 1, the incident field is

$$
B=\left[\begin{array}{lllllll}
0 & 0 & \operatorname{inc}_{f(k=3)}^{F_{1}} & \operatorname{inc}_{f(k=4)}^{F_{1}} & 0 & \cdots & \cdots
\end{array}\right]^{\mathrm{T}} .
$$

\section{References}

[1] Sewell E. Transmission of reverberant sound through a single leaf partition surrounded by an infinite rigid baffle. J Sound Vib 1970;12:21-32.

[2] Sharp B. Prediction methods for the sound transmission of building elements. Noise Control Eng 1978;11:53-63.

[3] London A. Transmission of reverberant sound through double walls. J Acoust Soc Am 1950;22:270-9.

[4] Beranek L, editor. Noise reduction. New York: McGraw-Hill; 1960.

[5] Fahy F. Foundations of engineering acoustics. New York: Academic Press; 2001.

[6] Fringuellino M, Guglielmone C. Progressive impedance method for the classical analysis of acoustic transmission loss in multilayered walls. Appl Acoust 2000;59:275-85.

[7] Bolton J, Shiau N, Kang Y. Sound transmission through multi-panel structures lined with elastic porous materials. J Sound Vib 1996;191(3):317-47.

[8] Cremer L, Heckl M, Ungar E. Structure-borne sound. Berlin: Springer; 1988.

[9] Vér I. Impact noise isolation of composite floors. J Acoust Soc Am 1970;50(4):1043-50.

[10] Heckl M, Rathe J. Relationship between the transmission loss and the impact-noise isolation of floor structures. J Acoust Soc Am 1963;35(11):1825-30.

[11] Tadeu A, António J. 2.5D Green functions for elastodynamic problems in layered acoustic and elastic formations. J Comp Model Eng Sci (CMES) 2002;2:477-95.

[12] Tadeu A, António J. Acoustic insulation of single panel walls provided by analytical expressions versus the mass law. J Sound Vib 2002;257(3):457-75.

[13] António J, Tadeu A, Godinho L. Analytical evaluation of the acoustic insulation provided by double infinite walls. J Sound Vib 2003;263:113-29.

[14] Graff K. Wave motion in elastic solids. New York: Dover; 1975.

[15] Acoubat Software, version 3.1.

[16] Standard ISO 140-1:1997(E). Acoustics-measurement of sound insulation in buildings and of building elements - Part 1: Requirements for laboratory test facilities with suppressed flanking transmission.

[17] Tadeu A, António J, Mateus D. Sound insulation provided by single and double panel walls. Appl Acoust 2004;65(1):15-29.

[18] Standard ISO 140-3: 1995(E). Acoustics-measurement of sound insulation in buildings and of building elements - Part 3: Laboratory measurements of airborne sound insulation of building elements.

[19] EN 12354-1: 2000 (E). Building acoustics-estimation of the acoustic performance of buildings from the performance of elements - Part 1: Airborne sound insulation between rooms.

[20] Documentation Technique, Imperalum; 2005. Available from: http://www.imperalum.com.

[21] ISO 140-8: 1997(E). Acoustics-measurement of sound insulation in buildings and of building elements - Part 8: Laboratory measurements of the reduction of transmitted impact noise by floor coverings on a heavyweight standard floor.

[22] EN 12354-2: Building acoustics-estimation of the acoustic performance of buildings from the performance of elements - Part 2: Impact sound insulation between rooms. 